\title{
LOCAL ENERGY ESTIMATE ON KERR BLACK HOLE BACKGROUNDS
}

\author{
DANIEL TATARU AND MIHAI TOHANEANU
}

\begin{abstract}
We study dispersive properties for the wave equation in the Kerr spacetime with small angular momentum. The main result of this paper is to establish uniform energy bounds and local energy decay for such backgrounds. This follows a similar result for the Schwarzschild space-time obtained in earlier work 21] by the authors and collaborators.
\end{abstract}

\section{INTRODUCTION}

The aim of this article is to study the decay properties of solutions to the wave equation in the Kerr space-time, which describes a rotating black hole. Until recently even the problem of obtaining uniform bounds for such solutions was completely open, and only some partial results were obtained in [14]. We also refer the reader to related independent work in [13. However, the techniques used in these papers are of a different flavor, as they do not carry out such a precise analysis of the dynamics near the trapped set.

Our aim here is to establish global in time energy bounds for the wave equation in the Kerr space-time, as well as a local energy decay estimate. These bounds apply in the full region outside the event horizon, as well as in a small neighborhood on the inside of the event horizon.

The starting point in our analysis is the earlier work [21] of the authors and collaborators, which establishes similar bounds for the wave equation in the Schwarzschild spacetime. The idea is to treat the Kerr geometry as a small perturbation of the Schwarzschild geometry, and then adapt the methods in [21]. Consequently in this article we are only considering Kerr black hole backgrounds with small angular momentum, which are close to the Schwarzschild space-time. Nevertheless, we are confident that our methods will carry over also to the case of large angular momentum.

Another goal of the earlier article [21] was to establish Strichartz estimates in the Schwarzschild space-time. We also aim to consider the similar problem for the Kerr space-time. However, this requires very different technical tools, and will be considered in a subsequent paper.

The local energy estimate in [21] is proved using the multiplier method; the delicate issue there is to show that a suitable multiplier can be found. This method is quite robust under small perturbations of the metric, and for the most part it easily carries over to the

Both authors were supported in part by NSF grant DMS0801261. 
Kerr backgrounds with small angular momentum. There is however one region where this does not apply, precisely near the photon sphere $r=3 M$ (which contains all 1 the trapped periodic geodesics in the Schwarzschild space-time). Hence most of the new analysis here is devoted to understanding what happens there.

The paper is organized as follows. In the next section we discuss the classical local energy decay decay estimates in the Minkowski space-time and small perturbation thereof. Then we provide a brief overview of the local energy estimates proved in [21] for the Schwarzschild space-time, along with a discussion of the relevant geometrical issues. Finally, the last section contains the description of the Kerr space-time and all the new results. Our main local energy estimate is contained in Theorem 4.1, This is complemented by higher order bounds in Theorem 4.4 .

\section{Local ENERGy DeCAY IN THE MinkoWski SPACE-TIME}

In the Minkowski space-time $\mathbb{R}^{3+1}$, consider the wave equation with constant coefficients

$$
\square u=f, \quad u(0)=u_{0}, \quad \partial_{t} u(0)=u_{1}
$$

Here $\square=\partial_{t}^{2}-\Delta$. More generally, let

$$
\square_{g}=\frac{1}{\sqrt{-g}} \partial_{i}\left(\sqrt{-g} g^{i j} \partial_{j}\right)
$$

be the usual d'Alembertian associated to a Lorentzian metric $g$.

The seminal estimate of Morawetz [19] asserts that for solutions to the homogeneous equation $\square u=0$ we have the estimate

$$
\int_{\mathbb{R}} \int_{\mathbb{R}^{3}} \frac{1}{|x|}|\not \nabla u|^{2}(t, x) d x d t+\int_{\mathbb{R}}|u(t, 0)|^{2} d t \lesssim\left\|\nabla u_{0}\right\|_{L^{2}}^{2}+\left\|u_{1}\right\|_{L^{2}}^{2}
$$

where $\not \nabla$ denotes the angular derivative. This is obtained combining energy estimates with the multiplier method. The radial multiplier $Q u=\left(\partial_{r}+\frac{1}{r}\right) u$ is used, where $r$ denotes the radial variable.

Within dyadic spatial regions one can control the full gradient $\nabla u$, but the square summability wth respect to dyadic scales is lost. Precisely, we define the $L^{2}$ local energy norm

$$
\|u\|_{L E_{M}}=\sup _{j \in \mathbb{Z}} 2^{-\frac{j}{2}}\|u\|_{L^{2}\left(\mathbb{R} \times\left\{|x| \in\left[2^{j-1}, 2^{j}\right]\right\}\right)}
$$

and its $H^{1}$ counterpart

$$
\|u\|_{L E_{M}^{1}}=\left\|\nabla_{x, t} u\right\|_{L E_{M}}+\left\||x|^{-1} u\right\|_{L E_{M}}
$$

For the inhomogeneous term we use the dual norm

$$
\|f\|_{L E_{M}^{*}}=\sum_{k \in \mathbb{Z}} 2^{\frac{k}{2}}\|f\|_{L^{2}\left(\mathbb{R} \times\left\{|x| \in\left[2^{k-1}, 2^{k}\right]\right\}\right)}
$$

\footnotetext{
1 except of course for the rays along the event horizon, which are not relevant to this discussion
} 
Then we have the following scale invariant local energy estimate for solutions $u$ to the inhomogeneous equation (2.1):

$$
\|\nabla u\|_{L_{t}^{\infty} L_{x}^{2}}+\|u\|_{L E_{M}^{1}} \lesssim\left\|\nabla u_{0}\right\|_{L^{2}}+\left\|u_{1}\right\|_{L^{2}}+\|f\|_{L E_{M}^{*}+L_{t}^{1} L_{x}^{2}}
$$

This is proved using a small variation of Morawetz's method, with multipliers of the form $a(r) \partial_{r}+b(r)$ where $a$ is positive, bounded and increasing.

There are many similar results obtained in the case of perturbations of the Minkowski space-time; see, for example, [19], [17], 16], [23], 24], [25], [2], [20]. Relevant to us is the case of small long range perturbations of the Minkowski space-time, considered in [22]. The metrics $g$ in $\mathbb{R}^{3+1}$ considered there satisfy

$$
\sum_{k \in \mathbb{Z}} \sup _{|x| \in\left[2^{k-1}, 2^{k}\right]}\left|g(t, x)-g_{M}\right|+|x|\left|\nabla_{x, t} g(t, x)\right|+|x|^{2}\left|\nabla_{x, t}^{2} g(t, x)\right| \leq \epsilon
$$

where $g_{M}$ stands for the Minkowski metric. Then as a special case of the results in [22] we have

Theorem 2.1. 22] Let $g$ be a Lorenzian metric in $\mathbb{R}^{3+1}$ which satisfies (2.7) with $\epsilon$ small enough. Then the solution $u$ to the inhomogeneous problem

$$
\square u=f, \quad u(0)=u_{0}, \quad \partial_{t} u(0)=u_{1}
$$

satisfies the estimate (2.7).

No general such results are known for large perturbations, where on one hand trapping for large frequencies and on the other hand eigenvalues and resonances for small frequencies create major difficulties. The Schwarzschild and Kerr metrics are such large perturbations where trapping plays a major role.

\section{LocAl ENERGy DECAY IN THE SCHWARZSCHILD SPACE-TIME}

In the original coordinates the Schwarzschild space-time is given as a metric whose line element is (for $\left.I=\mathbb{R} \times(2 M, \infty) \times \mathbb{S}^{2}\right)$

$$
d s^{2}=-\left(1-\frac{2 M}{r}\right) d t^{2}+\left(1-\frac{2 M}{r}\right)^{-1} d r^{2}+r^{2} d \omega^{2}
$$

where $d \omega^{2}$ is the measure on the sphere $\mathbb{S}^{2}$ and $t, r$ are the time, respectively the radius of the $\mathbb{S}^{2}$ spheres. This metric is well defined in two regions,

$$
I=\mathbb{R} \times(2 M, \infty) \times \mathbb{S}^{2}, \quad I I=\mathbb{R} \times(0,2 M) \times \mathbb{S}^{2}
$$

Let $\square_{S}$ denote the associated d'Alembertian.

The singularity at $r=0$ is a true metric singularity. However, the singularity at the event horizon $r=2 M$ is an apparent singularity that can be removed by a different choice of coordinates. Following [15], let

$$
r^{*}=r+2 M \log (r-2 M)-3 M-2 M \log M
$$

and let $v=t+r^{*}$. In the new coordinates $(r, v, \omega)$ the metric becomes

$$
d s^{2}=-\left(1-\frac{2 M}{r}\right) d v^{2}+2 d v d r+r^{2} d \omega^{2}
$$


and can be extended to a larger manifold $I \cup I I$. Moreover, if $w=t-r^{*}$, one can introduce global nonsingular coordinates (all the way to $r=0$ ) by rewriting the metric in the Kruskal-Szekeres coordinate system,

$$
v^{\prime}=e^{\frac{v}{4 M}}, \quad w^{\prime}=-e^{-\frac{w}{4 M}} .
$$

There are two places where trapping occurs on the Schwarzschild manifold. The first is at the event horizon $r=2 M$, where the trapped geodesics are the vertical ones in the $(r, v, \omega)$ coordinates. However, this family of trapped rays turns out to cause no difficulty in the decay estimates since the energy decays exponentially along it as $v \rightarrow \infty$. The second family of trapped rays occurs on the surface $r=3 M$ which is called the photon sphere. Null geodesics which are initially tangent to the photon sphere will remain on the surface for all times. Unlike the previous case, the energy is conserved for waves localized along such rays. However, what makes local energy decay estimates at all possible is the fact that the trapped rays on the photon sphere are hyperbolic.

The $(r, v, \omega)$ coordinates are nonsingular on the event horizon, but have the disadvantage that the level sets of $v$ are null surfaces. This is why it is more convenient to introduce

$$
\tilde{v}=v-\mu(r)
$$

where $\mu$ is a smooth function of $r$. In the $(\tilde{v}, r, \omega)$ coordinates the metric has the form

$$
\begin{aligned}
d s^{2}= & -\left(1-\frac{2 M}{r}\right) d \tilde{v}^{2}+2\left(1-\left(1-\frac{2 M}{r}\right) \mu^{\prime}(r)\right) d \tilde{v} d r \\
& +\left(2 \mu^{\prime}(r)-\left(1-\frac{2 M}{r}\right)\left(\mu^{\prime}(r)\right)^{2}\right) d r^{2}+r^{2} d \omega^{2} .
\end{aligned}
$$

On the function $\mu$ we impose the following two conditions:

(i) $\mu(r) \geq r^{*}$ for $r>2 M$, with equality for $r>5 M / 2$.

(ii) The surfaces $\tilde{v}=$ const are space-like, i.e.

$$
\mu^{\prime}(r)>0, \quad 2-\left(1-\frac{2 M}{r}\right) \mu^{\prime}(r)>0 .
$$

The first condition (i) insures that the $(r, \tilde{v}, \omega)$ coordinates coincide with the $(r, t, \omega)$ coordinates in $r>5 M / 2$. This is convenient but not required for any of our results. What is important is that in these coordinates the metric is asymptotically flat as $r \rightarrow \infty$ according to (2.7).

Given $0<r_{e}<2 M$ we consider the wave equation

$$
\square_{S} u=f
$$

in the cylindrical region

$$
\mathcal{M}_{R}=\left\{\tilde{v} \geq 0, r \geq r_{e}\right\}
$$

with initial data on the space-like surface

$$
\Sigma_{R}^{-}=\mathcal{M}_{R} \cap\{\tilde{v}=0\}
$$

The lateral boundary of $\mathcal{M}_{R}$,

$$
\Sigma_{R}^{+}=\mathcal{M}_{R} \cap\left\{r=r_{e}\right\}
$$


is also space-like, and can be thought of as the exit surface for all waves which cross the event horizon.

We define the initial (incoming) energy on $\Sigma_{R}^{-}$as

$$
E[u]\left(\Sigma_{R}^{-}\right)=\int_{\Sigma_{R}^{-}}\left(\left|\partial_{r} u\right|^{2}+\left|\partial_{\tilde{v}} u\right|^{2}+|\not \nabla u|^{2}\right) r^{2} d r d \omega
$$

the outgoing energy on $\Sigma_{R}^{+}$as

$$
E[u]\left(\Sigma_{R}^{+}\right)=\int_{\Sigma_{R}^{+}}\left(\left|\partial_{r} u\right|^{2}+\left|\partial_{\tilde{v}} u\right|^{2}+|\not \nabla u|^{2}\right) r_{e}^{2} d \tilde{v} d \omega
$$

and the energy on an arbitrary $\tilde{v}$ slice as

$$
E[u]\left(\tilde{v}_{0}\right)=\int_{\mathcal{M}_{R} \cap\left\{\tilde{v}=\tilde{v}_{0}\right\}}\left(\left|\partial_{r} u\right|^{2}+\left|\partial_{\tilde{v}} u\right|^{2}+|\not \nabla u|^{2}\right) r^{2} d r d \omega
$$

The choice of the local energy norm $L E_{S}$ is inspired from (2.3). However, there is a loss along the trapped geodesics on the photon sphere. Consequently, we introduce a modified $L^{2}$ local energy space

$$
\|u\|_{L E_{S}}=\left\|\left(1-\frac{3 M}{r}\right) u\right\|_{L E_{M}}
$$

and $H^{1}$ local energy space

$$
\|u\|_{L E_{S}^{1}}=\left\|\partial_{r} u\right\|_{L E_{M}}+\left\|\partial_{\tilde{v}} u\right\|_{L E_{S}}+\|\not \nabla u\|_{L E_{S}}+\left\|r^{-1} u\right\|_{L E_{M}}
$$

For the inhomogeneous term we use the norm

$$
\|f\|_{L E_{S}^{*}}=\left\|\left(1-\frac{3 M}{r}\right)^{-1} u\right\|_{L E_{M}}
$$

In the three formulas above we implicitely assume that all norms are restricted to the set $\mathcal{M}_{R}$ where we study the wave equation (3.2). Then we have the following result:

Theorem 3.1. 21] Let $u$ be so that $\square_{S} u=f$. Then we have

$$
E[u]\left(\Sigma_{R}^{+}\right)+\sup _{\tilde{v}} E[u](\tilde{v})+\|u\|_{L E_{S}^{1}}^{2} \lesssim E[u]\left(\Sigma_{R}^{-}\right)+\|f\|_{L E_{S}^{*}}^{2} .
$$

Note that, compared to the norms $L E_{M}, L E_{M}^{*}$, the weights have an additional polynomial singularity at $r=3 \mathrm{M}$, but there are no additional losses at the event horizon or near $\infty$. Furthermore, by more refined results in 21, this polynomial loss can be relaxed to a logarithmic loss, i.e. the factor $1-\frac{3 M}{r}$ can be improved to $|\ln (r-3 M)|^{-1}$ near $r=3 M$. This is related to the fact that the (periodic) trapped rays on the photon sphere are hyperbolic.

We also remark that in the expression of $L E_{S}^{1}$ it was sufficient to measure $\partial_{r} u$. This is due to the implicit cancelation caused by the fact that the symbol of the operator $\partial_{r}$ vanishes on the trapped set.

\footnotetext{
${ }^{2}$ notations are slightly changed compared to [21] in order to insure some uniformity across the three models described in the present paper
} 
The choice of $r_{e} \in(0,2 M)$ is unimportant since the $r$-slices $r=$ const $\in(0,2 M)$ are spacelike. Hence moving from one such $r$-slice to another is equivalent to solving a local hyperbolic problem, and involve no global considerations. Thus in the proof of the theorem one can assume without any restriction in generality that $r_{e}$ is close to $2 M$.

Local energy estimates were first proved in [18] for radially symmetric Schrödinger equations on Schwarzschild backgrounds. In [3, 4, 5, those estimates are extended to allow for general data for the wave equation. The same authors, in [6, 7], have provided studies that give certain improved estimates near the photon sphere $r=3 M$. Moreover, we note that variants of these bounds have played an important role in the works [8] and [11], 12] which prove analogues of the Morawetz conformal estimates on Schwarzschild backgrounds.

\section{LOCAL ENERGY DECAY IN THE KERR SPACE-TIME}

The Kerr geometry in Boyer-Lindquist coordinates is given by

$$
d s^{2}=g_{t t} d t^{2}+g_{t \phi} d t d \phi+g_{r r} d r^{2}+g_{\phi \phi} d \phi^{2}+g_{\theta \theta} d \theta^{2}
$$

where $t \in \mathbb{R}, r>0,(\phi, \theta)$ are the spherical coordinates on $\mathbb{S}^{2}$ and

$$
\begin{gathered}
g_{t t}=-\frac{\Delta-a^{2} \sin ^{2} \theta}{\rho^{2}}, \quad g_{t \phi}=-2 a \frac{2 M r \sin ^{2} \theta}{\rho^{2}}, \quad g_{r r}=\frac{\rho^{2}}{\Delta} \\
g_{\phi \phi}=\frac{\left(r^{2}+a^{2}\right)^{2}-a^{2} \Delta \sin ^{2} \theta}{\rho^{2}} \sin ^{2} \theta, \quad g_{\theta \theta}=\rho^{2}
\end{gathered}
$$

with

$$
\Delta=r^{2}-2 M r+a^{2}, \quad \rho^{2}=r^{2}+a^{2} \cos ^{2} \theta
$$

A straightforward computation gives us the inverse of the metric:

$$
\begin{gathered}
g^{t t}=-\frac{\left(r^{2}+a^{2}\right)^{2}-a^{2} \Delta \sin ^{2} \theta}{\rho^{2} \Delta}, \quad g^{t \phi}=-a \frac{2 M r}{\rho^{2} \Delta}, \quad g^{r r}=\frac{\Delta}{\rho^{2}}, \\
g^{\phi \phi}=\frac{\Delta-a^{2} \sin ^{2} \theta}{\rho^{2} \Delta \sin ^{2} \theta}, \quad g^{\theta \theta}=\frac{1}{\rho^{2}} .
\end{gathered}
$$

The case $a=0$ corresponds to the Schwarzschild space-time. We shall subsequently assume that $a$ is small $a \ll M$, so that the Kerr metric is a small perturbation of the Schwarzschild metric. We let $\square_{K}$ denote the d'Alembertian associated to the Kerr metric.

In the above coordinates the Kerr metric has singularities at $r=0$ on the equator $\theta=\pi / 2$ and at the roots of $\Delta$, namely $r_{ \pm}=M \pm \sqrt{M^{2}-a^{2}}$. As in the case of the Schwarzschild space, the singularity at $r=r_{+}$is just a coordinate singularity, and corresponds to the event horizon. The singularity at $r=r_{-}$is also a coordinate singularity; for a further discussion of its nature, which is not relevant for our results, we refer the reader to [10, 15]. To remove the singularities at $r=r_{ \pm}$we introduce functions $r^{*}, v_{+}$ and $\phi_{+}$so that (see [15])

$$
d r^{*}=\left(r^{2}+a^{2}\right) \Delta^{-1} d r, \quad d v_{+}=d t+d r^{*}, \quad d \phi_{+}=d \phi+a \Delta^{-1} d r .
$$


The metric then becomes

$$
\begin{aligned}
d s^{2}= & -\left(1-\frac{2 M r}{\rho^{2}}\right) d v_{+}^{2}+2 d r d v_{+}-4 a \rho^{-2} M r \sin ^{2} \theta d v_{+} d \phi_{+}-2 a \sin ^{2} \theta d r d \phi_{+}+\rho^{2} d \theta^{2} \\
& +\rho^{-2}\left[\left(r^{2}+a^{2}\right)^{2}-\Delta a^{2} \sin ^{2} \theta\right] \sin ^{2} \theta d \phi_{+}^{2}
\end{aligned}
$$

which is smooth and nondegenerate across the event horizon up to but not including $r=0$. Just like in [21, we introduce the function

$$
\tilde{v}=v_{+}-\mu(r)
$$

where $\mu$ is a smooth function of $r$. In the $\left(\tilde{v}, r, \phi_{+}, \theta\right)$ coordinates the metric has the form

$$
\begin{aligned}
d s^{2}= & \left(1-\frac{2 M r}{\rho^{2}}\right) d \tilde{v}^{2}+2\left(1-\left(1-\frac{2 M r}{\rho^{2}}\right) \mu^{\prime}(r)\right) d \tilde{v} d r \\
& -4 a \rho^{-2} M r \sin ^{2} \theta d \tilde{v} d \phi_{+}+\left(2 \mu^{\prime}(r)-\left(1-\frac{2 M r}{\rho^{2}}\right)\left(\mu^{\prime}(r)\right)^{2}\right) d r^{2} \\
& -2 a \theta\left(1+2 \rho^{-2} M r \mu^{\prime}(r)\right) \sin ^{2} d r d \phi_{+}+\rho^{2} d \theta^{2} \\
& +\rho^{-2}\left[\left(r^{2}+a^{2}\right)^{2}-\Delta a^{2} \sin ^{2} \theta\right] \sin ^{2} \theta d \phi_{+}^{2}
\end{aligned}
$$

On the function $\mu$ we impose the following two conditions:

(i) $\mu(r) \geq r^{*}$ for $r>2 M$, with equality for $r>5 M / 2$.

(ii) The surfaces $\tilde{v}=$ const are space-like, i.e.

$$
\mu^{\prime}(r)>0, \quad 2-\left(1-\frac{2 M r}{\rho^{2}}\right) \mu^{\prime}(r)>0 .
$$

As long as $a$ is small, we can work with the same function $\mu$ as in the case of the Schwarzschild space-time.

For convenience we also introduce

$$
\tilde{\phi}=\zeta(r) \phi_{+}+(1-\zeta(r)) \phi
$$

where $\zeta$ is a cutoff function supported near the event horizon and work in the $(\tilde{v}, r, \tilde{\phi}, \theta)$ coordinates which are identical to $(t, r, \phi, \theta)$ outside of a small neighborhood of the event horizon.

Carter [9] showed that the Hamiltonian flow is completely integrable by finding a fourth constant of motion $K$ that is preserved along geodesics. If $E$ and $L$ are the two constants of motion associated with the Killing vector fields $\partial_{t}$ and $\partial_{\phi}$, the equations for the null geodesics can be reduced to the following (see, for example, [10] or [26])

$$
\begin{aligned}
\rho^{2} \dot{t} & =a\left(L-E a \sin ^{2} \theta\right)+\frac{\left(r^{2}+a^{2}\right)\left(\left(r^{2}+a^{2}\right) E-a L\right)}{\Delta} \\
\rho^{2} \dot{\phi} & =\frac{L-E a \sin ^{2} \theta}{\sin ^{2} \theta}+\frac{\left(r^{2}+a^{2}\right) a E-a^{2} L}{\Delta} \\
\rho^{4} \dot{\theta}^{2} & =K-\frac{\left(L-E a \sin ^{2} \theta\right)^{2}}{\sin ^{2} \theta} \\
\rho^{4} \dot{r}^{2} & =-K \Delta+\left(\left(r^{2}+a^{2}\right) E-a L\right)^{2}
\end{aligned}
$$

where the overdot denotes differentiation with respect to an affine parameter $s$. This parametrization of the null geodesics is nondegenerate away from the surfaces $r=r_{ \pm}$. 
Next we discuss the geometry of the trapped null geodesics. The level sets $r=r_{0}$ of $r$ are time-like for $r_{0}>r_{+}$, null for $r=r_{+}$and space-like for $r_{-}<r_{0}<r_{+}$. The latter implies that there are no trapped null geodesics inside the region $\left\{r_{-}<r<r_{+}\right\}$. On the null surfaces $r=r_{ \pm}$, through each point there is a unique null vector which is tangent and which generates a trapped null geodesics.

To find the trapped null geodesics in the region $r>r_{+}$it suffices to consider the behavior of the fourth degree polynomial

$$
P(r)=-K \Delta+\left(\left(r^{2}+a^{2}\right) E-a L\right)^{2}
$$

in the last equation in (4.1). At least one of the parameters $E, K$ and $L$ should be nonzero, and the third equation shows that $K \geq 0$ and that we cannot simultaneously have $E=K=0$. Thus $P$ is always nondegenerate. The key observation is that that the simple zeroes of $P$ correspond to turning points in the last equation, and only the double zeroes are steady states. There are several cases to consider.

a) If $E=0$ then $K>0$. Thus $P$ has at most one positive root, where it changes sign from + to - . This root is a right turning point for the ode, and there are no trapped null geodesics.

b) $E \neq 0$. Then $P$ has degree 4 and $P \geq 0$ in $\left[r_{-}, r_{+}\right]$. If $P$ has any zero in $\left[r_{-}, r_{+}\right]$ then the square expression must vanish, and this zero must be a double zero. We claim that in $\left(r_{+}, \infty\right) P$ has either no root or two roots (counted with multiplicity); this is easily seen, as $P$ must have either (at least) two complex conjugate roots or a negative root (the sum of the roots equals 0 ) and (at least) another one smaller than $r_{-}$(since $\left.P\left(r_{-}\right) \geq 0\right)$. There are three subcases:

b1) $P$ has no roots larger than $r_{+}$. Then $r$ is monotone along null geodesics, and there are no trapped null geodesics.

b2) $P$ has two distinct positive roots $r_{+}<r_{1}<r_{2}$. There it must change sign from + to - , respectively from - to + . Hence $r_{1}$ is a right turning point and $r_{2}$ is a left turning point for the ode. Thus no trapped null geodesics exist.

b3) $P$ has a double positive real root $r_{0}$. Then this root is a steady state, and all other solutions converge to the steady state at one end, and escape to 0 or infinity at the other end.

This analysis shows that the only trapped null geodesics are those along which $r$ is constant. The polynomial $P$ has a double root if the following two relations hold,

$$
\left(\left(r^{2}+a^{2}\right) E-a L\right)^{2}=K \Delta, \quad 2 r E\left(\left(r^{2}+a^{2}\right) E-a L\right)=K(r-M)
$$

which we rewrite in the form

$$
K=\frac{r^{2} E^{2} \Delta}{(r-M)^{2}}, \quad a L=E\left(r^{2}+a^{2}-\frac{2 r \Delta}{r-M}\right)
$$

The right hand side in the $\dot{\theta}$ equation must be nonnegative. Substituting in the above two relations we obtain a necessary condition for the existence of trapped geodesics, namely the inequality

$$
\left(2 r \Delta-(r-M) \rho^{2}\right)^{2} \leq 4 a^{2} r^{2} \Delta \sin ^{2} \theta
$$


One can show that this condition is also sufficient. The expression on the left has the form

$$
2 r \Delta-(r-M) \rho^{2}=r^{2}(r-3 M)+2 r a^{2}-(r-M) a^{2} \cos ^{2} \theta
$$

If $a=0$ then it has a single positive nondegenerate zero at $r=3 M$, which is the photon sphere in the Schwarzschild metric. Hence if $0<a \ll M$ it will still have a single zero which is close to $3 M$. A rough computation leads to a bound of the form

$$
|r-3 M| \leq 2 a, \quad a \ll 2 M
$$

Thus all trapped null geodesics lie within $O(a)$ of the $r=3 M$ sphere.

We would like a characterization of the aforementioned trapped geodesics in the phase space. Let $\tau, \xi, \Phi$ and $\Theta$ be the Fourier variables corresponding to $t, r, \phi$ and $\theta$, and

$$
p(r, \phi, \tau, \xi, \Phi, \Theta)=g^{t t} \tau^{2}+2 g^{t \phi} \tau \Phi+g^{\phi \phi} \Phi^{2}+g^{r r} \xi^{2}+g^{\theta \theta} \Theta^{2}
$$

be the principal symbol of $\square_{K}$. On any null geodesic one has

$$
p(t, r, \phi, \theta, \tau, \xi, \Phi, \Theta)=0 .
$$

Moreover, the Hamilton flow equations give us

$$
\begin{gathered}
\dot{r}=-\frac{\partial p}{\partial \xi}=-\frac{2 \Delta}{\rho^{2}} \xi \\
\dot{\xi}=\frac{\partial p}{\partial r}=g_{, r}^{t t} \tau^{2}+2 g_{, r}^{t \phi} \tau \Phi+g_{, r}^{\phi \phi} \Phi^{2}+g_{, r}^{r r} \xi^{2}+g_{, r}^{\theta \theta} \Theta^{2}
\end{gathered}
$$

We rewrite the latter in the form

$$
\rho^{2} \dot{\xi}=\rho^{2} \frac{\partial p}{\partial r}=-2 R_{a}(r, \tau, \Phi) \Delta^{-2}+\rho^{2} \partial_{r}\left(\rho^{-2}\right) p+2(r-M) \xi^{2}
$$

where

$$
R_{a}(r, \tau, \Phi)=\left(r^{2}+a^{2}\right)\left(r^{3}-3 M r^{2}+a^{2} r+a^{2} M\right) \tau^{2}-2 a M\left(r^{2}-a^{2}\right) \tau \Phi-a^{2}(r-M) \Phi^{2}
$$

For geodesics with constant $r$, one needs to impose the additional condition $\dot{r}=0$. Hence from (4.5) either $r=r_{ \pm}$, which correspond to the geodesics at $r=2 M$ in the Schwarzschild case, or $\xi=0$. In the latter case from (4.7) we obtain a polynomial equation for $r$, namely

$$
R_{a}(r, \tau, \Phi)=0
$$

Furthermore, due to (4.4) we must also have the inequality

$$
-\left(\left(r^{2}+a^{2}\right)^{2}-a^{2} \Delta \sin ^{2} \theta\right) \tau^{2}-2 a M r \tau \Phi+\frac{\Delta-a^{2} \sin ^{2} \theta}{\sin ^{2} \theta} \Phi^{2} \leq 0
$$

If $a$ is small and $r$ is as in (4.3) this allows us to bound $\Phi$ in terms of $\tau$,

$$
|\Phi| \leq 4 M|\tau|
$$

For $\Phi$ in this range and small $a$ the polynomial $\tau^{-2} R_{a}(r, \tau, \Phi)$ can be viewed as a small perturbation of

$$
\tau^{-2} R_{0}(r, \tau, \Phi)=r^{4}(r-3 M)
$$


which has a simple root at $r=3 M$. Hence for small $a$ the polynomial $R_{a}$ has a simple root close to $3 M$, which we denote by $r_{a}(\tau, \phi)$. By homogeneity considerations and the implicit function theorem we can further express $r_{a}$ in the form

$$
r_{a}(\tau, \Phi)=3 M \tilde{r}\left(\frac{a}{M}, \frac{\Phi}{M \tau}\right), \quad \tilde{r} \in C^{\infty}([-\epsilon, \epsilon] \times[-4,4])
$$

Since $r_{0}(\tau, \Phi)=3 M$, it follows that we can write $r_{a}(\tau, \Phi)$ in the form

$$
r_{a}(\tau, \Phi)=3 M+a F\left(\frac{a}{M}, \frac{\Phi}{M \tau}\right), \quad F \in C^{\infty}([-\epsilon, \epsilon] \times[-4,4])
$$

The above analysis shows that the trapped null geodesics corresponding to frequencies $(\tau, \Phi)$ are located at radial frequency $\xi=0$ and position $r=r_{a}(\tau, \xi)$. One would be naively led to define the local smoothing spaces associated to the Kerr space-time by replacing the factor $r-3 M$ in (3.9) and (3.11) with the modified factor $r-r_{a}(\tau, \Phi)$. Unfortunately, this is no longer a scalar function, but a symbol of a pseudodifferential operator. In addition, this operator depends on the time Fourier variable $\tau$, which is inconvenient for energy estimates on time $(\tilde{v})$ slabs.

Consequently, we replace the $r-r_{a}(\tau, \Phi)$ weight with a polynomial in $\tau$ which has the same symbol on the characteristic set $p=0$. More precisely, for $r$ close to $3 M$ we factor

$$
p(r, \phi, \tau, \xi, \Phi, \Theta)=g^{t t}\left(\tau-\tau_{1}(r, \phi, \xi, \Phi, \Theta)\right)\left(\tau-\tau_{2}(r, \phi, \xi, \Phi, \Theta)\right)
$$

where $\tau_{1}, \tau_{2}$ are real distinct smooth 1-homogeneous symbols. On the cone $\tau=\tau_{i}$ the symbol $r-r_{a}(\tau, \phi)$ equals

$$
c_{i}(r, \phi, \xi, \Phi, \Theta)=r-r_{a}\left(\tau_{i}, \Phi\right)=r-3 M-a F\left(\frac{a}{M}, \frac{\Phi}{M \tau_{i}}\right), \quad i=1,2
$$

If $r$ is close to $3 M$ and $|a| \ll M$ then on the characteristic set of $p$ we have $|\phi|<4 M|\tau|$, therefore the symbols $c_{i}$ are well defined, smooth and homogeneous. They are also nonzero outside an $O(a)$ neighborhood of $3 M$.

We use the symbols $c_{i}$ to define associated microlocally weighted function spaces $L_{c_{i}}^{2}$ in a neighborhood $I \times \mathbb{S}^{2}$ of $3 M \times \mathbb{S}^{2}$ which does not depend on $a$ for small $a$. For functions $u$ supported in $I \times \mathbb{S}^{2}$ we set

$$
\|u\|_{L_{c_{i}}^{2}}^{2}=\left\|c_{i}(D, x) u\right\|_{L^{2}}^{2}+\|u\|_{H^{-1}}^{2}
$$

There is an ambiguity in this notation as we have not specified the coordinate frame in which we view $c_{i}$ as a pseudodifferential operator. However, it is easy to see that different frames lead to equivalent norms. We note that the low frequencies in $c_{i}$ are also irrelevant, and can be removed with a suitable cutoff. After removing the low frequencies, the quantization that we use for $c_{i}$ becomes unimportant as well. We also define a dual norm $c_{i} L^{2}$ for functions $g$ supported in $I \times \mathbb{S}^{2}$, namely

$$
\|g\|_{c_{i} L^{2}}^{2}=\inf _{c_{i}(x, D) g_{1}+g_{2}=g}\left(\left\|g_{1}\right\|_{L^{2}}^{2}+\left\|g_{2}\right\|_{H^{1}}^{2}\right)
$$

Since the symbols $c_{i}$ are nonzero outside an $O(a)$ neighborhood of $3 M$, it follows that both norms $L_{c_{i}}^{2}$ and $c_{i} L^{2}$ are equivalent to $L^{2}$ outside a similar neighborhood. 
Now we can define local smoothing norms associated to the Kerr space-time. Let $\chi(r)$ be a smooth cutoff function which is supported in the above neighborhood $I$ of $3 M$ and which equals 1 near $3 M$. Then we set

$$
\begin{aligned}
\|u\|_{L E_{K}^{1}}= & \left\|\chi\left(D_{t}-\tau_{2}(D, x)\right) \chi u\right\|_{L_{c_{1}}^{2}}+\left\|\chi\left(D_{t}-\tau_{1}(D, x)\right) \chi u\right\|_{L_{c_{2}}^{2}}+\left\|\left(1-\chi^{2}\right) \partial_{t} u\right\|_{L E_{M}} \\
& +\left\|\left(1-\chi^{2}\right) \not \nabla u\right\|_{L E_{M}}+\left\|\partial_{r} u\right\|_{L E_{M}}+\left\|r^{-1} u\right\|_{L E_{M}}
\end{aligned}
$$

We remark that this norm is equivalent to the Minkowski norm $L E_{M}^{1}$ outside an $O(a)$ neighborhood of $3 M$, but it is degenerate on the trapped set.

For the nonhomogeneous term in the equation we define a dual structure,

$$
\|f\|_{L E_{K}^{*}}=\|(1-\chi) f\|_{L E_{M}^{*}}+\|\chi f\|_{c_{1} L^{2}+c_{2} L^{2}}
$$

To state the main result of this paper we use the notations in (3.3)-(3.8), with the parameter $r_{e}$ chosen so that $r_{-}<r_{e}<r_{+}$:

Theorem 4.1. Let $u$ solve $\square_{K} u=f$ in $\mathcal{M}_{R}$. Then

$$
\|u\|_{L E_{K}^{1}}^{2}+\sup _{\tilde{v}} E[u](\tilde{v})+E[u]\left(\Sigma_{R}^{+}\right) \lesssim E[u]\left(\Sigma_{R}^{-}\right)+\|f\|_{L E_{K}^{*}}^{2} .
$$

in the sense that the left hand side is finite and the inequality holds whenever the right hand side is finite.

The proof of the result uses the multiplier method. Part of the difficulty is caused by the fact that, as shown in [1, there is no differential multiplier that provides us with a positive local energy norm. What we do instead is find a suitable pseudodifferential operator that does the job. This is chosen so that its symbol vanishes on trapped rays, which leads to a local energy norm which is degenerate there.

As in the Schwarzschild case, the choice of $r_{e} \in\left(r_{-}, r_{+}\right)$is unimportant since the $r$-slices $r=$ const $\in\left(r_{-}, r_{+}\right)$are spacelike. Hence in the proof of the theorem one can assume without any restriction in generality that $r_{e}$ is close to $r_{+}$.

Proof. The theorem is proved using a modification of the arguments in 21]. Let us first quickly recall the key steps in the proof of Theorem 3.1 as in [21]. We begin with the energy-momentum tensor

$$
Q_{\alpha \beta}[u]=\partial_{\alpha} u \partial_{\beta} u-\frac{1}{2} g_{\alpha \beta} \partial^{\gamma} u \partial_{\gamma} u
$$

Its contraction with respect to a vector field $X$ is denoted by

$$
P_{\alpha}[u, X]=Q_{\alpha \beta}[u] X^{\beta}
$$

and its divergence is

$$
\nabla^{\alpha} P_{\alpha}[u, X]=\square_{g} u \cdot X u+\frac{1}{2} Q_{\alpha \beta}[u] \pi^{\alpha \beta}
$$

where $\pi^{\alpha \beta}$ is the deformation tensor of $X$, given by

$$
\pi_{\alpha \beta}=\nabla_{\alpha} X_{\beta}+\nabla_{\beta} X_{\alpha}
$$

A special role is played by the Killing vector field

$$
K=\partial_{\tilde{v}}
$$


whose deformation tensor is zero.

Integrating the above divergence relation for a suitable choice of $X$ does not suffice in order to prove the local energy estimates, as in general the deformation tensor can only be made positive modulo a Lagrangian term of the form $q \partial^{\alpha} u \partial_{\alpha} u$. Hence some lower order corrections are required. For a vector field $X$, a scalar function $q$ and a 1 -form $m$ we define

$$
P_{\alpha}[u, X, q, m]=P_{\alpha}[u, X]+q u \partial_{\alpha} u-\frac{1}{2} \partial_{\alpha} q u^{2}+\frac{1}{2} m_{\alpha} u^{2} .
$$

The divergence formula gives

$$
\nabla^{\alpha} P_{\alpha}[u, X, q, m]=\square_{g} u(X u+q u)+Q[u, X, q, m],
$$

where

$$
Q[u, X, q, m]=\frac{1}{2} Q_{\alpha \beta}[u] \pi^{\alpha \beta}+q \partial^{\alpha} u \partial_{\alpha} u+m_{\alpha} u \partial^{\alpha} u+\left(\nabla^{\alpha} m_{\alpha}-\frac{1}{2} \nabla^{\alpha} \partial_{\alpha} q\right) u^{2} .
$$

So far these computations apply both for the Schwarzschild and the Kerr metrics. From here one, we will use the sub(super)scripts $S$, respectively $K$ to indicate when a computation is perform with respect to one metric or another.

To prove the local energy decay in the Schwarzschild space-time, $X, q$ and $m$ are chosen as in the following lemma:

Lemma 4.2. There exist a smooth vector field

$$
X=b(r)\left(1-\frac{3 M}{r}\right) \partial_{r}+c(r) K
$$

with $c$ supported near the event horizon and $b>0$ bounded so that

$$
\left|\partial_{r}^{\alpha} b\right| \leq c_{\alpha} r^{-\alpha}
$$

a smooth function $q(r)$ with

$$
\left|\partial_{r}^{\alpha} q\right| \leq c_{\alpha} r^{-1-\alpha}
$$

and a smooth 1-form $m$ supported near the event horizon $r=2 M$ so that

(i) The quadratic form $Q^{S}[u, X, q, m]$ is positive definite,

$$
Q^{S}[u, X, q, m] \gtrsim r^{-2}\left|\partial_{r} u\right|^{2}+\left(1-\frac{3 M}{r}\right)^{2}\left(r^{-2}\left|\partial_{\tilde{v}} u\right|^{2}+r^{-1}|\not \nabla u|^{2}\right)+r^{-4} u^{2} .
$$

(ii) $X(2 M)$ points toward the black hole, $X(d r)(2 M)<0$, and $\langle m, d r\rangle(2 M)>0$.

The local energy estimate is obtained by integrating the divergence relation (4.12) with $X+C K$ instead of $X$, where $C$ is a large constant, on the domain

$$
D=\left\{0<\tilde{v}<\tilde{v}_{0}, r>r_{e}\right\}
$$

with respect to the measure induced by the metric, $d V_{S}=r^{2} d r d \tilde{v} d \omega$. This yields

$$
\int_{D} Q^{S}[u, X, q, m] d V_{S}=-\int_{D} \square_{S} u((X+C K) u+q u) d V_{S}+B D R^{S}[u]
$$

where $B D R^{S}[u]$ denotes the boundary terms

$$
B D R^{S}[u]=\left.\int\langle d \tilde{v}, P[u, X+C K, q, m]\rangle r^{2} d r d \omega\right|_{\tilde{v}=0} ^{\tilde{v}=\tilde{v}_{0}}-\int\langle d r, P[u, X+C K, q, m]\rangle r_{e}^{2} d \tilde{v} d \omega
$$


Using the condition (ii) in the Lemma and Hardy type inequalities, it is shown in 21] that for large $C$ and $r_{e}$ close to $2 M$ the boundary terms have the correct sign,

$$
B D R^{S}[u] \leq c_{1} E[u]\left(\Sigma_{R}^{-}\right)-c_{2}\left(E[u]\left(\tilde{v}_{0}\right)+E[u]\left(\Sigma_{R}^{+}\right)\right), \quad c_{1}, c_{2}>0
$$

Consequently, by applying the Cauchy-Schwartz inequality for the first term on the right of (4.14) we obtain a slightly weaker form of the local energy estimate (3.12), namely

$$
E[u]\left(\Sigma_{R}^{+}\right)+\sup _{\tilde{v}} E[u](\tilde{v})+\|u\|_{L E W_{S}^{1}}^{2} \lesssim E[u]\left(\Sigma_{R}^{-}\right)+\|f\|_{L E W_{S}^{*}}^{2} \cdot
$$

where the weaker norm $L E W_{S}^{1}$ and the stronger norm $L E W_{S}^{*}$ are defined by

$$
\|u\|_{L E W_{S}^{1}}^{2}=\int_{\mathcal{M}_{R}}\left(r^{-2}\left|\partial_{r} u\right|^{2}+\left(1-\frac{3 M}{r}\right)^{2}\left(r^{-2}\left|\partial_{\tilde{v}} u\right|^{2}+r^{-1}|\not \nabla u|^{2}\right)+r^{-4} u^{2}\right) r^{2} d r d \tilde{v} d \omega
$$

respectively

$$
\|f\|_{L E W_{S}^{*}}^{2}=\int_{\mathcal{M}_{R}} r^{2}\left(1-\frac{3 M}{r}\right)^{-2} f^{2} r^{2} d r d \tilde{v} d \omega
$$

These norms are equivalent with the stronger norms $L E_{S}^{1}$, respectively $L E_{S}^{*}$ for $r$ in a bounded set. On the other hand for large $r$ the Schwarzschild space can be viewed as a small perturbation of the Minkovski space. Thus the transition from (4.16) to (3.12) is achieved in [21] by cutting away a bounded region and then using a perturbation of a Minkowski space estimate. This part of the proof translates without any changes to the case of the Kerr space-time. Our goal in what follows will be to establish the Kerr counterpart of (4.16), namely

$$
E[u]\left(\Sigma_{R}^{+}\right)+\sup _{\tilde{v}} E[u](\tilde{v})+\|u\|_{L E W_{K}^{1}}^{2} \lesssim E[u]\left(\Sigma_{R}^{-}\right)+\|f\|_{L E W_{K}^{*}}^{2} \cdot
$$

where the norms $L E W_{K}^{1}$, respectively $L E W_{K}^{*}$ coincide with $L E_{K}^{1}$, respectively $L E_{K}^{*}$ for bounded $r$, and with $L E W_{S}^{1}$, respectively $L E W_{S}^{*}$ for large $r$. More precisely, if $\chi(r)$ is a smooth compactly supported cutoff function which equals 1 say for $r<4 M$ then we set

$$
\|u\|_{L E W_{K}^{1}}^{2}=\|\chi u\|_{L E_{K}^{1}}^{2}+\|(1-\chi) u\|_{L E W_{S}^{1}}^{2}
$$

respectively

$$
\|u\|_{L E W_{K}^{*}}^{2}=\|\chi u\|_{L E_{K}^{*}}^{2}+\|(1-\chi) u\|_{L E W_{S}^{*}}^{2}
$$

Different choices for $\chi$ lead to different but equivalent norms.

It is useful to first consider the effect of the same multiplier in the Kerr metric. The two metrics are close when measured in the same euclidean frame $x=r \omega$ with $r \geq r_{e}$. Precisely, with $\partial$ standing for $\partial_{t}$ and $\partial_{x}, x=r \omega$,

$$
\left|\partial^{\alpha}\left[\left(g_{K}\right)_{i j}-\left(g_{S}\right)_{i j}\right]\right| \leq c_{\alpha} \frac{a}{r^{2+|\alpha|}}, \quad\left|\partial^{\alpha}\left[\left(g_{K}\right)^{i j}-\left(g_{S}\right)^{i j}\right]\right| \leq c_{\alpha} \frac{a}{r^{2+|\alpha|}}
$$

From this and the size and regularity properties of $X, q$ and $m$ it follows that

$$
\left|P_{\alpha}^{S}[u, X, q, m]-P_{\alpha}^{K}[u, X, q, m]\right| \lesssim \frac{a}{r^{2}}|\nabla u|^{2}
$$

respectively

$$
\left|Q^{S}[u, X, q, m]-Q^{K}[u, X, q, m]\right| \lesssim a\left(\frac{1}{r^{2}}|\nabla u|^{2}+\frac{1}{r^{4}}|u|^{2}\right)
$$


Hence integrating the divergence relation (4.12) in the Kerr space-time over the same domain $D$ but with respect to the Kerr induced measure $d V_{K}=\rho^{2} d r d \tilde{v} d \omega$ we obtain

$$
\int_{D} Q^{K}[u, X, q, m] d V_{K}=-\int_{D} \square_{K} u((X+C K) u+q u) d V_{K}+B D R^{K}[u]
$$

The bound (4.19) shows that for small $a$ the boundary terms retain their positivity properties in (4.15), namely

$$
B D R^{K}[u] \leq c_{1} E[u]\left(\Sigma_{R}^{-}\right)-c_{2}\left(E[u]\left(\tilde{v}_{0}\right)+E[u]\left(\Sigma_{R}^{+}\right)\right), \quad c_{1}, c_{2}>0
$$

However, 4.20) merely shows that

$$
Q_{K}[u, X, q, m] \gtrsim r^{-2}\left|\partial_{r} u\right|^{2}+\left[\left(1-\frac{3 M}{r}\right)^{2}-C a\right]\left(r^{-2}\left|\partial_{\tilde{v}} u\right|^{2}+r^{-1}|\not \nabla u|^{2}\right)+r^{-4} u^{2}
$$

and the right hand side is no longer positive definite near $r=3 M$. Thus we cannot close the argument as in the Schwarzschild case. As shown in [1], changing the vector field $X$ near $r=3 M$ would not help.

To remedy this, we need to use a pseudodifferential modification $S$ of the vector field $X$. We will choose $S$ so that its kernel is supported in a small neighborhood of $(3 M, 3 M)$; this insures that there will be no additional contributions at $r=r_{e}$. Furthermore, in order to be able to carry out the computations near the initial and final surfaces $\tilde{v}=0, \tilde{v}_{0}$ we take $S$ to be a first order differential operator with respect to $\tilde{v}$. Similarly, we modify the Lagrangian factor $q$ using a pseudodifferential correction $E$, which is also a first order differential operator with respect to $\tilde{v}$.

We also need to choose a quantization which is consistent with the Kerr measure. Here we have a few choices which have equivalent results. For our selection we use euclideanlike coordinates $x=\omega r$. Given a real symbol $s$ its euclidean Weyl quantization $s^{w}$ is selfadjoint with respect to the euclidean measure $d V=r^{2} d r d \omega$. However, in our case we need to work with the Kerr induced measure $d V_{K}=\rho^{2} d r d \omega$. Hence we slightly abuse notations and redefine the Weyl quantization as

$$
s^{w}:=\frac{r}{\rho} s^{w} \frac{\rho}{r}
$$

If $s$ is a real symbol, then $s^{w}$ (re)defined above is a selfadjoint operator in $L^{2}\left(d V_{K}\right)$.

Another issue which does not affect our analysis but needs to be addressed is that we are using pseudodifferential operators in an exterior domain $\left\{r>r_{e}\right\}$ and some care must be given to what happens near $r=r_{e}$. To keep things simple, in what follows all operators we work with are compactly supported in the sense that their kernels are supported away from $r_{e}$ and infinity; even better, supported in a small neighborhood of $3 M$.

In what follows we consider a skewadjoint pseudodifferential operator $S$ and a selfadjoint pseudodifferential operator $E$ of the form 3

$$
S=i s_{1}^{w}+s_{0}^{w} \partial_{t}, \quad E=e_{0}^{w}+\frac{1}{i} e_{-1} \partial_{t}
$$

\footnotetext{
${ }^{3}$ Since we are away from the event horizon the variable $\tilde{v}$ coincides with $t$. We make this substitution here and later.
} 
where $s_{1} \in S^{1}, s_{0}, e_{0} \in S^{0}$ and $e_{-1} \in S^{-1}$ are real symbols, homogeneous outside a neighborhood of 0 . Commuting and integrating by parts we obtain the counterpart of the relation (4.21), namely

$$
I Q^{K}[u, S, E]=-\Re \int_{D} \square_{K} u \cdot(S+E) u d V_{K}+B D R^{K}[u, S, E]
$$

Here $B D R^{K}[u, S, E]$ represents the boundary terms obtained in the integration by parts with respect to $t$. Its exact form is not important, as all we need to use here is the bound

$$
\left|B D R^{K}[u, S, E]\right| \lesssim E[u](0)+E[u]\left(\tilde{v}_{0}\right)
$$

We note that due to the presence of the cutoff function $\chi$ in both operators $S$ and $E$ there are no contributions on the lateral boundary $r=r_{e}$.

On the other hand $I Q^{K}[u, S, E]$ represents a quadratic form in $\left(u, D_{t} u\right)$ which can be written in the form

$$
I Q^{K}[u, S, E]=\int_{D} Q_{2}^{w} u \cdot \bar{u}+2 \Re Q_{1}^{w} u \cdot \overline{D_{t} u}+Q_{0}^{w} D_{t} u \overline{D_{t} u} d V_{K}
$$

where $Q_{j}^{w} \in O P S^{j}$ are selfadjoint pseudodifferential operators so that

$$
Q_{2}^{w}+2 Q_{1}^{w} D_{t}+Q_{0}^{w} D_{t}^{2}=\frac{1}{2}\left(\left[\square_{K}, S\right]+\square_{K} E+E \square_{K}\right)
$$

We remark that for arbitrary operators $S$ and $E$ the expression above on the right is in general a third order differential operator in $t$. However, the operator $S$ will always be chosen so that the coefficient of $D_{t}^{3}$ vanishes. We define the principal symbol of the quadratic form $I Q^{K}[u, S, E]$ as

$$
q^{K}[S, E]=q_{2}+2 q_{1} \tau+q_{0} \tau^{2}
$$

The previous relation shows that it satisfies

$$
q^{K}[S, E]=\frac{1}{2 i}\{p, s\}+p e \quad \bmod S^{0}+\tau S^{-1}+\tau^{2} S^{-2}
$$

We add (4.21) with $a$ times (4.24). The boundary terms are estimated by (4.22) and (4.25). Using the duality between the spaces $c_{i} L^{2}$ and $L_{c_{i}}^{2}$ we can also estimate

$$
\left|\int_{D} f(X+C K+q+a(S+E)) u d V_{K}\right| \lesssim\|f\|_{L E W_{K}^{*}}\|u\|_{L E W_{K}^{1}}
$$

Hence in order to prove (4.17) it would suffice to show that the symbols $s$ and $e$ can be chosen so that

$$
\int_{D} Q^{K}[u, X, q, m] d V_{K}+a I Q^{K}[u, S, E] \gtrsim\|u\|_{L E W_{K}^{1}}^{2}
$$

Here we aim to choose $S$ and $E$ uniformly with respect to small $a$. In effect, our construction below yields symbols $s$ and $e$ which are analytic with respect to $a$. We remark that the choice of $S$ and $E$ is only important in the region where $r$ is close to $3 M$. Outside this region, $Q^{K}[u, X, q, m]$ is already positive definite and the contribution of $a I Q^{K}[u, S, E]$ is negligible.

We consider first the expression $Q^{K}[u, X, q, m]$. Near $r=3 M$ this has the form

$$
Q^{K}[u, X, q, m]=\sum q^{K, \alpha \beta} \partial_{\alpha} u \partial_{\beta} u+q^{K, 0} u^{2}
$$


where its principal symbol $q^{K}=q^{K, \alpha \beta} \eta_{\alpha} \eta_{\beta}$ and the lower order coefficient $q^{K, 0}$ are given by the relation

$$
q^{K}=\frac{1}{2 i}\{p, X\}+q p, \quad q^{K, 0}=-\frac{1}{2} \nabla^{\alpha} \partial_{\alpha} q
$$

We do not need to exactly compute the above expression in the Kerr case, but it is useful to perform the computation in the simpler case of the Schwarzschild space. There we have

$$
p=-\left(1-\frac{2 M}{r}\right)^{-1} \tau^{2}+\left(1-\frac{2 M}{r}\right) \xi^{2}+\frac{1}{r^{2}} \lambda^{2}, \quad X=i b(r)\left(1-\frac{3 M}{r}\right) \xi
$$

where $\lambda$ stands for the spherical Fourier variable. Hence we obtain

$$
\begin{aligned}
r^{2} q^{S} & =\frac{1}{2 i}\left\{r^{2} p, X\right\}+\left(q-r^{-1} b(r)(r-3 M)\right)\left(r^{2} p\right) \\
& =\alpha_{S}^{2}(r) \tau^{2}+\beta_{S}^{2}(r) \xi^{2}+\tilde{q}(r)\left(r^{2} p\right)
\end{aligned}
$$

where, near $r=3 M$,

$$
\begin{gathered}
\alpha_{S}^{2}(r)=\frac{r b(r)(r-3 M)^{2}}{(r-2 M)^{2}} \\
\beta_{S}^{2}(r)=\frac{3 M}{r^{2}} b\left(r^{2}-2 M r\right)+\left(1-\frac{3 M}{r}\right)\left(b^{\prime}\left(r^{2}-2 M r\right)-b(r-M)\right)
\end{gathered}
$$

respectively

$$
\tilde{q}(r)=q-r^{-1} b(r)(r-3 M) .
$$

Here we have used the fact that $b>0$ to write the first two coefficients as squares.

For our choice of $q$ and $r$ we know that the relation (4.13) holds. This implies that the following two inequalities must hold:

$$
q^{S} \gtrsim \xi^{2}+(r-3 M)^{2}\left(\tau^{2}+\lambda^{2}\right), \quad q^{S, 0}>0
$$

Given the form of $q^{S}$, the first relation implies that $\tilde{q}$ is a multiple of $(r-3 M)^{2}$, and that in addition there is a smooth function $\nu(r)$ so that

$$
\frac{r^{3}}{r-2 M} \tilde{q}=\nu(r) \alpha_{S}^{2}(r), \quad 0<\nu<1
$$

This allows us to obtain the following sum of squares representation for $q^{S}$ :

$r^{2} q^{S}=(1-\nu(r)) \alpha_{S}^{2}(r) \tau^{2}+\beta^{2}(r) \xi^{2}+\nu_{1}(r) \alpha_{S}^{2}(r)\left(\lambda^{2}+\left(r^{2}-2 r M\right) \xi^{2}\right), \quad \nu_{1}=\frac{r-2 M}{r^{3}} \nu$

The symbol $\lambda^{2}$ of the spherical Laplacian ca also be written as sums of squares of differential symbols,

$$
\lambda^{2}=\lambda_{1}^{2}+\lambda_{2}^{2}+\lambda_{3}^{2}
$$

where in Euclidean coordinates we can write

$$
\left\{\lambda_{1}, \lambda_{2}, \lambda_{3}\right\}=\left\{x_{i} \eta_{j}-x_{j} \eta_{i}, i \neq j\right\}
$$

$$
r^{2} q^{S}=(1-\nu(r)) \alpha_{S}^{2}(r) \tau^{2}+\beta_{S}^{2}(r) \xi^{2}+\nu_{1}(r) \alpha_{S}^{2}(r)\left(\lambda_{1}^{2}+\lambda_{2}^{2}+\lambda_{3}^{2}+\left(r^{2}-2 r M\right) \xi^{2}\right)
$$


We return now to the question of finding symbols $s$ and $e$ so that the bound (4.28) holds. Near $r=3 M$, the principal symbol of the quadratic form on the left in (4.28) is

$$
\frac{1}{2 i}\{p, X+a s\}+p(q+a e)
$$

In order to prove (4.28) at the very least we would like the above symbol to be nonnegative, and to satisfy the bound

$$
\frac{1}{2 i}\{p, X+a s\}+p(q+a e) \gtrsim c_{2}^{2}\left(\tau-\tau_{1}\right)^{2}+c_{1}^{2}\left(\tau-\tau_{2}\right)^{2}+\xi^{2}
$$

However, such a bound would not a-priori suffice since translating it to operator bounds would require using the Fefferman-Phong inequality, which does not hold in general for systems. Hence we prove a more precise result, and show that the symbols $s$ and $e$ can be chosen so that we have a favorable sum of squares representation for the above expression, which extends the sum of squares (4.33) to $a \neq 0$.

Lemma 4.3. Let a be sufficiently small. Then there exist smooth homogeneous symbols $s \in S_{\text {hom }}^{1}, e \in S_{\text {hom }}^{0}$, also depending smoothly in a, so that for $r$ close to $3 M$ we have sum of squares representation

$$
\rho^{2}\left(\frac{1}{2 i}\{p, X+a s\}+p(q+a e)\right)=\sum_{j=1}^{8} \mu_{j}^{2}
$$

where $\mu_{j} \in S_{\text {hom }}^{1}+\tau S_{\text {hom }}^{0}$ satisfy the following properties:

(i) The decomposition (4.34) extends the decomposition (4.33) in the sense that

$$
\begin{aligned}
\left(\mu_{1}, \mu_{2}, \mu_{3}, \mu_{4}, \mu_{5}, \mu_{6}\right)= & \left((1-\nu)^{\frac{1}{2}} \alpha_{S} \tau, \beta_{S} \xi, \nu_{1}^{\frac{1}{2}} \alpha_{S} \lambda_{1}, \nu_{1}^{\frac{1}{2}} \alpha_{S} \lambda_{2}, \nu_{1}^{\frac{1}{2}} \alpha_{S} \lambda_{3}, \nu_{1}^{\frac{1}{2}} \alpha_{S} \xi\right) \\
& \bmod a\left(S_{\text {hom }}^{1}+\tau S_{\text {hom }}^{0}\right)
\end{aligned}
$$

and

$$
\left(\mu_{7}, \mu_{8}\right) \in \sqrt{a}\left(S_{h o m}^{1}+\tau S_{h o m}^{0}\right)
$$

(ii) The family of symbols $\left\{\mu_{j}\right\}_{j=1,6}$ is elliptically equivalent with the family of symbols $\left(c_{2}\left(\tau-\tau_{1}\right), c_{1}\left(\tau-\tau_{2}\right), \xi\right)$ in the sense that we have a representation of the form

$$
\mu=M v, \quad v=\left(\begin{array}{c}
c_{2}\left(\tau-\tau_{1}\right) \\
c_{1}\left(\tau-\tau_{2}\right) \\
\xi
\end{array}\right)
$$

where the symbol valued matrix $M \in M^{8 \times 3}\left(S_{\text {hom }}^{0}\right)$ has maximum rank 3 everywhere.

Proof. Setting $\tilde{q}=q-2\{\ln \rho, X\}$ respectively $\tilde{e}=e-2\{\ln \rho, s\}$ we compute

$$
\rho^{2}\left(\frac{1}{2 i}\{p, X+a s\}+(q+a e) p\right)=\frac{1}{2 i}\left\{\rho^{2} p, X+a s\right\}+(\tilde{q}+a \tilde{e})\left(\rho^{2} p\right)
$$

We first choose the symbol $s$ so that the Poisson bracket $\left\{\rho^{2} p, X+a s\right\}$ has the correct behavior on the characteristic set $p=0$. Recall that the symbol of $X$ is $i r^{-1} b(r)(r-3 M) \xi$, where the vanishing coefficient at $3 M$ corresponds exactly to the location of the trapped rays. Its natural counterpart in the Kerr space-time is the symbol

$$
\tilde{s}(r, \tau, \xi, \Phi)=i r^{-1} b(r)\left(r-r_{0}(\tau, \Phi)\right) \xi .
$$


This coincides with $X$ in the Schwarzschild case $a=0$, and it is well defined and smooth in $a$ for $r$ near $3 M$ and $|\Phi|<4|\tau|$. In particular it is well defined in a neighborhood of the characteristic set $p=0$, which is all we use in the sequel.

We use (4.7) to compute the Poisson bracket

$$
\begin{aligned}
\frac{1}{i}\left\{\rho^{2} p, \tilde{s}\right\}= & -\left(\rho^{2} p\right)_{r} r^{-1} b(r)\left(r-r_{0}(\tau, \Phi)\right)+\xi\left(\rho^{2} p\right)_{\xi} \partial_{r}\left(r^{-1} b(r)\left(r-r_{0}(\tau, \Phi)\right)\right) \\
= & 2 r^{-1} b(r) R(r, \tau, \Phi) \Delta^{-2}\left(r-r_{0}(\tau, \Phi)\right) \\
& +\left[2 \Delta \partial_{r}\left(r^{-1} b(r)\left(r-r_{0}(\tau, \Phi)\right)\right)-2(r-M) r^{-1} b(r)\left(r-r_{0}(\tau, \Phi)\right)\right] \xi^{2}
\end{aligned}
$$

Since $r_{0}(\tau, \Phi)$ is the unique zero of $R(r, \tau, \Phi)$ near $r=3 M$ and is close to $3 M$, it follows that we can write

$$
\frac{1}{2 i}\left\{\rho^{2} p, \tilde{s}\right\}=\alpha^{2}(r, \tau, \Phi) \tau^{2}\left(r-r_{0}(\tau, \Phi)\right)^{2}+\beta^{2}(r, \tau, \Phi) \xi^{2} \quad \text { on } \quad\{p=0\}
$$

where $\alpha, \beta \in S_{\text {hom }}^{0}$ are positive symbols. We note that in the Schwarzschild case the symbols $\alpha$ and $\beta$ are simply functions of $r$, see the first two terms in (4.30).

Unfortunately $\tilde{s}$ is not a polynomial in $\tau$, which limits its direct usefulness. To remedy that we first note that

$$
\tilde{s}-\left(i r^{-1} b(r)(r-3 M) \xi\right) \in a S_{h o m}^{1}
$$

Hence by (the simplest form of) the Malgrange preparation theorem we can write

$$
\frac{1}{i} \tilde{s}=(r-3 M) b(r) \xi+a\left(s_{1}(r, \xi, \theta, \Theta, \Phi)+s_{0}(r, \xi, \theta, \Theta, \Phi) \tau\right)+a \gamma(\tau, r, \xi, \theta, \Theta, \Phi) p
$$

with $s_{1} \in S_{h o m}^{1}, s_{0} \in S_{h o m}^{0}$ and $\gamma \in S_{h o m}^{-1}$. Then we define the desired symbol $s$ by

$$
s=s_{1}+s_{0} \tau
$$

The Poisson bracket $\frac{1}{i}\left\{\rho^{2} p, s\right\}$ is a third degree polynomial in $\tau$. Hence, after division by $p=-g^{t t}\left(\tau-\tau_{1}\right)\left(\tau-\tau_{2}\right)$, taking also (4.33) into account, we can write

$$
\frac{1}{2 i}\left\{\rho^{2} p, X+a s\right\}+\tilde{q}\left(\rho^{2} p\right)=\gamma_{2}+\gamma_{1} \tau+\left[e_{S}+a\left(e_{0}+e_{-1} \tau\right)\right]\left(\tau-\tau_{1}\right)\left(\tau-\tau_{2}\right) .
$$

where, by (4.33), the coefficient $e_{S}$ corresponding to the Schwarzschild case is given by

$$
e_{S}=(1-\nu(r)) \alpha^{2}(r)
$$

It remains to show that the right hand side of (4.36) can be expressed as a sum of squares as in the lemma modulo an error $a\left(S_{\text {hom }}^{0}+\tau S_{h o m}^{-1}\right) p$. Note that the symbols $e_{0}$ and $e_{-1}$ play no role in this, as they can be included in the error.

The coefficients $\gamma_{1}$ and $\gamma_{2}$ can be computed using the relation (4.35) and the fact that $\left\{\rho^{2} p, X+a s\right\}=\left\{\rho^{2} p, \tilde{s}\right\}$ on $p=0$ (i.e. when $\tau=\tau_{i}$ ). We denote

$$
\alpha_{i}=\frac{2\left|\tau_{i}\right|}{\tau_{1}-\tau_{2}} \alpha\left(r, \tau_{i}, \Phi\right)\left(r-r_{0}\left(\tau_{i}, \Phi\right)\right) \in S_{\text {hom }}^{0}, \quad \beta_{i}=\beta\left(r, \tau_{i}, \Phi\right),
$$

observing that $\alpha_{i}$ can be used as substitutes for the $c_{i}$ 's in the lemma since they are elliptic multiples of $c_{i}$. Then we have

$$
\frac{1}{2 i}\left\{\rho^{2} p, \tilde{s}\right\}\left(\tau_{i}\right)=\frac{1}{4} \alpha_{i}^{2}\left(\tau_{1}-\tau_{2}\right)^{2}+\beta_{i}^{2} \xi^{2}
$$


which gives the following expressions for $\gamma_{1}, \gamma_{2}$ :

$$
\gamma_{2}=\frac{1}{4}\left(\tau_{1}-\tau_{2}\right)\left(\alpha_{2}^{2} \tau_{1}-\alpha_{1}^{2} \tau_{2}\right)+\frac{\tau_{1} \beta_{2}^{2}-\tau_{2} \beta_{1}^{2}}{\tau_{1}-\tau_{2}} \xi^{2}, \quad \gamma_{1}=\frac{1}{4}\left(\tau_{1}-\tau_{2}\right)\left(\alpha_{1}^{2}-\alpha_{2}^{2}\right)+\frac{\beta_{1}^{2}-\beta_{2}^{2}}{\tau_{1}-\tau_{2}} \xi^{2}
$$

We use the first components of $\gamma_{1}$ and $\gamma_{2}$ to obtain a sum of squares as follows:

$$
\begin{aligned}
\left(\tau_{1}-\tau_{2}\right)\left(\alpha_{2}^{2} \tau_{1}-\alpha_{1}^{2} \tau_{2}\right)+\tau\left(\tau_{1}-\tau_{2}\right)\left(\alpha_{1}^{2}-\alpha_{2}^{2}\right) & =\nu\left(\alpha_{1}\left(\tau-\tau_{2}\right)-\alpha_{2}\left(\tau-\tau_{1}\right)\right)^{2} \\
& +(1-\nu)\left(\alpha_{1}\left(\tau-\tau_{2}\right)+\alpha_{2}\left(\tau-\tau_{1}\right)\right)^{2} \\
& -4 e_{K}\left(\tau-\tau_{1}\right)\left(\tau-\tau_{2}\right)
\end{aligned}
$$

where

$$
e_{K}=\frac{\left(\alpha_{1}-\alpha_{2}\right)^{2}}{4}+(1-\nu) \alpha_{1} \alpha_{2}
$$

We remark that in the Schwarzschild case we have $\tau_{2}=-\tau_{1}$ and also $\alpha_{1}=\alpha_{2}=\alpha_{S}$ and $\beta_{1}=\beta_{2}=\beta_{S}$. In particular this shows that

$$
e_{K}-e_{S} \in a\left(S_{h o m}^{0}+\tau S_{h o m}^{-1}\right)
$$

which accounts for the $e_{S}$ factor in (4.36). It remains to consider the $\xi^{2}$ terms in (4.37). This is easier since the coefficients $\beta_{1}, \beta_{2}$ are positive and have a small difference $\beta_{1}-\beta_{2} \in$ $a S_{\text {hom }}^{0}$. Precisely, for a large $C$ we can write

$$
\begin{aligned}
\frac{\tau_{1} \beta_{2}^{2}-\tau_{2} \beta_{1}^{2}}{\tau_{1}-\tau_{2}}+\tau \frac{\beta_{1}^{2}-\beta_{2}^{2}}{\tau_{1}-\tau_{2}}= & \frac{1}{2}\left(\beta_{1}^{2}+\beta_{2}^{2}-C a\right)+\frac{\left(C a-\beta_{2}^{2}+\beta_{1}^{2}\right)\left(\tau-\tau_{2}\right)^{2}}{2\left(\tau_{1}-\tau_{2}\right)^{2}} \\
& +\frac{\left(C a-\beta_{1}^{2}+\beta_{2}^{2}\right)\left(\tau-\tau_{1}\right)^{2}}{2\left(\tau_{1}-\tau_{2}\right)^{2}}+O(a) p
\end{aligned}
$$

Summing this with (4.38) we obtain the desired sums of squares representation,

$$
\begin{aligned}
\frac{1}{2 i}\left\{\rho^{2} p, X+a s\right\}+\left(\rho^{2} p\right) \tilde{q} \in & \frac{\nu}{4}\left(\alpha_{1}\left(\tau-\tau_{2}\right)-\alpha_{2}\left(\tau-\tau_{1}\right)\right)^{2} \\
& +\frac{1-\nu}{4}\left(\alpha_{1}\left(\tau-\tau_{2}\right)+\alpha_{2}\left(\tau-\tau_{1}\right)\right)^{2}+\frac{1}{2}\left(\beta_{1}^{2}+\beta_{2}^{2}-C a\right) \xi^{2} \\
& +\frac{\left(C a-\beta_{2}^{2}+\beta_{1}^{2}\right)\left(\tau-\tau_{2}\right)^{2}}{2\left(\tau_{1}-\tau_{2}\right)^{2}} \xi^{2}+\frac{\left(C a-\beta_{1}^{2}+\beta_{2}^{2}\right)\left(\tau-\tau_{1}\right)^{2}}{2\left(\tau_{1}-\tau_{2}\right)^{2}} \xi^{2} \\
& +a\left(S_{\text {hom }}^{0}+S_{h o m}^{-1} \tau\right)\left(\tau-\tau_{1}\right)\left(\tau-\tau_{2}\right)
\end{aligned}
$$

Then $e$ is chosen so that the last term accounts for the contribution of $\tilde{e}$.

Part (ii) of the lemma directly follows. For part (i) we still need to specify which are the symbols $\mu_{j}$. Precisely, we set

$$
\begin{gathered}
\mu_{1}^{2}=\frac{1-\nu}{4}\left(\alpha_{1}\left(\tau-\tau_{2}\right)+\alpha_{2}\left(\tau-\tau_{1}\right)\right)^{2}, \quad \mu_{2}^{2}=\frac{1}{2}\left(\beta_{1}^{2}+\beta_{2}^{2}-C a\right) \xi^{2} \\
\mu_{7}^{2}=\frac{\left(C a-\beta_{2}^{2}+\beta_{1}^{2}\right)\left(\tau-\tau_{2}\right)^{2}}{2\left(\tau_{1}-\tau_{2}\right)^{2}} \xi^{2}, \quad \mu_{8}^{2}=\frac{\left(C a-\beta_{1}^{2}+\beta_{2}^{2}\right)\left(\tau-\tau_{1}\right)^{2}}{2\left(\tau_{1}-\tau_{2}\right)^{2}} \xi^{2}
\end{gathered}
$$

Finally for $\mu_{3,4,5}$ and $\mu_{6}$ we set

$$
\mu_{3,4,5}^{2}=\frac{\lambda_{1,2,3}^{2}}{\lambda^{2}+\left(r^{2}-2 r M\right) \xi^{2}} \frac{\nu}{4}\left(\alpha_{1}\left(\tau-\tau_{2}\right)-\alpha_{2}\left(\tau-\tau_{1}\right)\right)^{2},
$$


respectively

$$
\mu_{6}^{2}=\frac{\left(r^{2}-2 r M\right) \xi^{2}}{\lambda^{2}+\left(r^{2}-2 r M\right) \xi^{2}} \frac{\nu}{4}\left(\alpha_{1}\left(\tau-\tau_{2}\right)-\alpha_{2}\left(\tau-\tau_{1}\right)\right)^{2}
$$

It is easy to see that for $a=0$ these symbols coincide with the coresponding Schwarzschild symbols. The proof of the lemma is concluded.

In what follows we use the above lemma to prove the bound (4.28) and conclude the proof of the theorem. We begin with symbols $s$ and $e$ as in the lemma. These are homogeneous symbols, and we can make them smooth by truncating away the low frequencies. They are only defined near $r=3 M$, therefore some spatial truncation is also necessary. Let $\chi$ be a smooth cutoff function supported near $3 M$ which equals 1 in a neighborhood of $3 M$, chosen so that we have a smooth partition of unity in $r$,

$$
1=\chi^{2}(r)+\chi_{o}^{2}(r)
$$

At first we define the truncated operators

$$
\tilde{S}=\chi s^{w} \chi, \quad \tilde{E}=\chi e^{w} \chi
$$

This choice would yield an expression $Q^{K}[u, \tilde{S}, \tilde{E}]$ with a principal symbol

$$
q_{\text {princ }}^{K}[\tilde{S}, \tilde{E}]=\chi^{2}\left(\frac{1}{2 i}\{p, s\}+p e\right)+\frac{1}{i} \chi s\{p, \chi\}
$$

For these choices of $\tilde{S}$ and $\tilde{E}$ we consider the expression $I Q^{K}[u, \tilde{S}, \tilde{E}]$ which is given by (4.26) with $Q_{2}^{w}, Q_{1}^{w}$ and $Q_{0}^{w}$ as in (4.27). We observe that in general we can only say that the right hand side of (4.27) is of the form

$$
\frac{1}{2}\left(\left[\square_{K}, \tilde{S}\right]+\square_{K} \tilde{E}+\tilde{E} \square_{K}\right)=Q_{2}^{w}+2 Q_{1}^{w} D_{t}+Q_{0}^{w} D_{t}^{2}+Q_{-1}^{w} D_{t}^{3}, \quad Q_{j}^{w} \in O P S^{j}
$$

However, its principal symbol $q_{\text {princ }}^{K}[\tilde{S}, \tilde{E}]$ is at most a second order polynomial in $\tau$. Hence by the Weyl calculus we can write

$\frac{1}{2}\left(\left[\square_{K}, \tilde{S}\right]+\square_{K} \tilde{E}+\tilde{E} \square_{K}\right)-\left(q_{p r i n c}^{K}[\tilde{S}, \tilde{E}]\right)^{w} \in O P S^{0}+O P S^{-1} D_{t}+O P S^{-2} D_{t}^{2}+O P S^{-3} D_{t}^{3}$

In particular this shows that $Q_{-1}^{w} \in O P S^{-3}$. To eliminate this term we slightly adjust our choice of $\tilde{E}$ to

$$
\tilde{E}=\chi e^{w} \chi-e_{a u x}^{w} D_{t}
$$

where the operator $e_{\text {aux }}^{w}$ is chosen so that

$$
g^{t t} e_{\text {aux }}^{w}+e_{\text {aux }}^{w} g^{t t}=Q_{-1}^{w}
$$

This is possible since the coefficient $g^{t t}$ of $\tau^{2}$ in $p$ is a scalar function which is nonzero near $r=3 M$. Also as defined $e_{a u x}^{w} \in O P S^{-3}$ and has kernel supported near $r=3 M$.

Having insured that the $D_{t}^{3}$ term does not appear, we divide $I Q^{K}[u, \tilde{S}, \tilde{E}]$ into two parts,

$$
I Q^{K}[u, \tilde{S}, \tilde{E}]=I Q_{\text {princ }}^{K}[u, \tilde{S}, \tilde{E}]+I Q_{\text {aux }}^{K}[u, \tilde{S}, \tilde{E}]
$$

where the main component is given by

$$
I Q_{\text {princ }}^{K}[u, \tilde{S}, \tilde{E}]=\int_{D} Q_{2, p}^{w} u \cdot \bar{u}+2 \Re Q_{1, p}^{w} u \cdot \overline{D_{t} u}+Q_{0, p}^{w} D_{t} u \overline{D_{t} u} d V_{K}
$$


with operators $Q_{2, p}^{w}, Q_{1, p}^{w}$ and $Q_{0, p}^{w}$ defined by

$$
Q_{2, p}^{w}+2 Q_{1, p}^{w} D_{t}+Q_{0, p}^{w} D_{t}^{2}=\chi\left(\frac{1}{2 i}\{p, s\}+p e\right)^{w} \chi
$$

while the remainder is given by a similar expression with operators $Q_{2, a}^{w}, Q_{1, a}^{w}$ and $Q_{0, a}^{w}$ whose principal symbols are supported away from $r=3 M$. More precisely, we have

$$
Q_{2, a}^{w}+2 Q_{1, a}^{w} D_{t}+Q_{0, a}^{w} D_{t}^{2}-\left(\frac{1}{i} \chi s\{p, \chi\}\right)^{w} \in O P S^{0}+O P S^{-1} D_{t}+O P S^{-2} D_{t}^{2}
$$

Hence, using the fact that the $L E W_{K}^{1}$ norm is nondegenerate outside an $O(a)$ neighborhood of $3 M$ we can bound in an elliptic fashion

$$
\left|I Q_{a u x}^{K}[u, \tilde{S}, \tilde{E}]\right| \lesssim\|u\|_{L E W_{K}^{1}}^{2}+\left\|D_{t} u\right\|_{H_{\text {comp }}^{-1}}^{2}
$$

where the last term on the right represents the $H^{-1}$ norm of $D_{t} u$ in a compact region in $r$ (precisely, a neighborhood of $3 M$ ).

In order to conclude the proof of the theorem we turn our attention to the bound (4.28), which we seek to establish with $S$ and $E$ replaced with $\tilde{S}$, respectively $\tilde{E}$. We will show that

$$
\int_{D} Q^{K}[u, X, q, m] d V_{K}+a I Q_{p r i n c}^{K}[u, \tilde{S}, \tilde{E}] \gtrsim\|u\|_{L E W_{K}^{1}}^{2}-O(a)\left\|D_{t} u\right\|_{H_{c o m p}^{-1}}^{2}
$$

We decompose the left hand side of (4.41) into an outer part and an inner part,

$$
L H S(4.41)=L H S \text { (4.41) }_{\text {out }}+L H S \text { (4.41) }_{\text {in }}
$$

where

$$
\begin{aligned}
L H S \text { (4.41) }_{\text {out }} & =\int_{D} \chi_{o}^{2} Q^{K}[u, X, q, m] d V_{k} \\
L H S \text { (4.41) }_{\text {in }} & =\int_{D} \chi^{2} Q^{K}[u, X, q, m] d V_{k}+a I Q_{\text {princ }}^{K}[u, \tilde{S}, \tilde{E}]
\end{aligned}
$$

For the first part we use the pointwise positivity of $Q^{K}$ away from $3 M$ (see (4.23) to conclude that

$$
\text { LHS (4.41) }{ }_{\text {out }} \gtrsim \int_{D} \chi_{o}^{2}\left(r^{-2}|\nabla u|^{2}+r^{-4}|u|^{2}\right) d V_{K}
$$

The second part is a quadratic form which for convenience we fully recall here (see 44.29) and (4.39):

$$
\begin{aligned}
L H S(4.41)_{i n}= & \int_{D} \chi^{2}\left(q^{K, \alpha \beta} \partial_{\alpha} u \partial_{\beta} u+q^{K, 0} u^{2}\right) d V^{K} \\
& +a \int_{D} Q_{2, p}^{w} u \cdot \bar{u}+2 \Re Q_{1, p}^{w} u \cdot \overline{D_{t} u}+Q_{0, p}^{w} D_{t} u \overline{D_{t} u} d V_{K}
\end{aligned}
$$

where the coefficients $q^{K, \alpha \beta}, q^{K, 0}$ and operators $Q_{j, p}^{w}$ satisfy

respectively

$$
q^{K, \alpha \beta} \eta_{\alpha} \eta_{\beta}=\frac{1}{2 i}\{p, X\}+q p, \quad q^{K, 0}>0
$$

$$
Q_{2, p}^{w}+2 Q_{1, p}^{w} D_{t}+Q_{0, p}^{w} D_{t}^{2}=\chi\left(\frac{1}{2 i}\{p, s\}+p e\right)^{w} \chi
$$


We carefully observe that in the two parts of the expression for $L H S(4.41$ in ine cutoff function $\chi$ appears in different places. In the first part, it is applied before the differentiation, while in the second part it is applied before the pseudodifferential operator. It does not make much sense to commute at this point. In the first part, we would produce lower order terms which may significantly alter $q^{K, 0}$. In the second part, we would lose the compact support of the kernels for the operators $Q_{j, p}^{w}$.

Since $s$ and $e$ are chosen as in Lemma 4.3, it follows that the principal symbol for $L H S(4.41)_{i n}$ admits the sum of squares representation (4.34). We want to translate this into a sum of squares decomposition for $L H S(4.41)_{i n}$. However, some care is required due to the different positions of the cutoff $\chi$, as explained above. The symbols $\mu_{k}=\mu_{k}(a)$ are in general of pseudodifferential type. However, part (i) of the Lemma guarantees that in the Schwarzschild case they are of differential type. Consequently, we write

$$
\mu_{k}(a)=\mu_{k}(0)+\mu_{k}(a)-\mu_{k}(0)
$$

and use this decomposition to define the pseudodifferential operators

$$
M_{k}=\chi \mu_{k}(0)(x, D)+\left(\mu_{k}(a)-\mu_{k}(0)\right)^{w} \chi
$$

Then using the Weyl calculus it follows that for $L H S$ (4.41) in $_{\text {in }}$ we have the representation $L H S$ (4.41) $_{\text {in }}=\int_{D} \sum_{k}\left|M_{k} u\right|^{2}+q^{K, 0} \chi^{2} u^{2} d V_{k}+\int_{D} R_{2}^{w} u \cdot \bar{u}+2 \Re R_{1}^{w} D_{t} u \cdot \bar{u}+R_{0}^{w} D_{t} u \cdot \overline{D_{t} u} d V_{K}$

where the remainder terms satisfy $r_{j} \in a S^{j-2}$. What is important here is that the remainder is of size $O(a)$. This follows from our choice of the operators $M_{k}$, which guarantees that when $a=0$ the remainder is zero.

Combining the last relation with (4.42) we obtain the bound

$$
\int_{D} \chi_{o}^{2} r^{-2}|\nabla u|^{2}+r^{-4}|u|^{2}+\sum_{k}\left|M_{k} u\right|^{2} d V_{K} \lesssim L H S(4.41)+a\left(\|u\|_{L_{\text {comp }}^{2}}^{2}+\left\|D_{t} u\right\|_{H_{\text {comp }}^{-1}}^{2}\right)
$$

where the last two terms on the right account for the remainder terms involving the operators $R_{j}^{w}$, which can be bounded using norms of $u$ and $D_{t} u$ in a compact region in $r$, away from $r=0$ and $r=\infty$.

It is easy to see that the above left hand side dominates $\|u\|_{L E W_{K}^{1}}$. For $r$ away one uses only the first two terms. On the other hand for $r$ close to $3 M$ we use part (iii) of the Lemma, which guarantees that the symbols $c_{1}\left(\tau-\tau_{2}\right), c_{2}\left(\tau-\tau_{1}\right)$ and $\xi$ can be recovered in an elliptic fashion from the principal symbols $\mu_{k}$ of $M_{k}$. Thus (4.41) is proved. Together with (4.40) this shows that

$$
\|u\|_{L E W_{K}^{1}}^{2} \lesssim \int_{D} Q^{K}[u, X, q, m] d V_{K}+a I Q^{K}[u, \tilde{S}, \tilde{E}]+O(a)\left\|D_{t} u\right\|_{H_{\text {comp }}^{-1}}^{2}
$$

The final step in the proof of (4.28) is to establish that the last error term above is negligible. We can account for it in an elliptic manner. Precisely, for any compactly supported selfadjoint operator $Q \in O P S^{-1}$ we can use $Q^{2}$ in a Lagrangian term and integrate by parts (commute) to obtain

$$
\begin{gathered}
2 \Re \int_{D}\left(g^{t t}\right)^{-1} \square_{K} u \cdot \overline{Q^{2} u} d V_{K}=\left\|Q D_{t} u\right\|_{L^{2}}^{2}+O\left(\left\|Q D_{t} u\right\|_{L^{2}}\|u\|_{L_{\text {comp }}^{2}}^{2}+\|u\|_{L_{\text {comp }}^{2}}^{2}\right. \\
\left.+E[u](0)+E[u]\left(\tilde{v}_{0}\right)\right)
\end{gathered}
$$


which leads to the elliptic bound

$$
\left\|Q D_{t} u\right\|_{L^{2}}^{2} \lesssim\|u\|_{L_{\text {comp }}^{2}}^{2}+\left\|\square_{K} u\right\|_{H_{\text {comp }}^{-1}}^{2}+E[u](0)+E[u]\left(\tilde{v}_{0}\right)
$$

and further to

$$
\left\|D_{t} u\right\|_{H_{c o m p}^{-1}}^{2} \lesssim\|u\|_{L_{\text {comp }}^{2}}^{2}+\left\|\square_{K} u\right\|_{H_{\text {comp }}^{-1}}^{2}+E[u](0)+E[u]\left(\tilde{v}_{0}\right)
$$

Thus (4.28) follows, and the proof of the theorem is concluded.

Note that Theorem 4.1 tells us, in particular, that if we start with an initial data $\left(u_{0}, u_{1}\right) \in H^{1} \times L^{2}$ then $u(\tilde{v}) \in H^{1}$ is uniformly bounded for all $\tilde{v}>0$. A natural question to ask is if this is also true for higher $H^{n}$ norms. For $n \geq 1$ we define

$$
\|u\|_{L E_{K}^{n+1}}=\sum_{|\alpha| \leq n}\left\|\partial^{\alpha} u\right\|_{L E_{K}^{1}}
$$

respectively

$$
\|f\|_{L E_{K}^{n *}}=\sum_{|\alpha| \leq n}\left\|\partial^{\alpha} f\right\|_{L E_{K}^{*}}
$$

The higher order energies are similarly defined,

$$
E^{n+1}[u]\left(\Sigma_{R}^{ \pm}\right)=\sum_{|\alpha| \leq n} E\left[\partial^{\alpha} u\right]\left(\Sigma_{R}^{ \pm}\right), \quad E^{n+1}[u]\left(\tilde{v}_{0}\right)=\sum_{|\alpha| \leq n} E\left[\partial^{\alpha} u\right]\left(\tilde{v}_{0}\right)
$$

We then have the following

Theorem 4.4. Let $n$ be a positive integer and $u$ satisfy $\square_{K} u=f$ with initial data $\left(u_{0}, u_{1}\right) \in H^{n+1} \times H^{n}$ on $\Sigma_{R}^{-}$and $f \in L E_{K}^{n *}\left(\mathcal{M}_{R}\right)$. Then

$$
E^{n+1}[u]\left(\Sigma_{R}^{+}\right)+\sup _{\tilde{v}>0} E^{n+1}[u]\left(\tilde{v}_{0}\right)+\|u\|_{L E_{K}^{n+1}}^{2} \lesssim\left\|u_{0}\right\|_{H^{n+1}}^{2}+\left\|u_{1}\right\|_{H^{n}}^{2}+\|f\|_{L E_{K}^{n *}}^{2}
$$

Proof. We remark that by trace regularity results we have

$$
\sum_{|\alpha| \leq n-1}\left\|\partial^{\alpha} f\right\|_{L^{2}\left(\Sigma_{R}^{-}\right)} \lesssim\|f\|_{L E_{K}^{n *}}
$$

Since the initial surface $\Sigma_{R}^{-}$is space-like, we can use the equation to derive all higher $\tilde{v}$ derivatives of $u$ in terms of the Cauchy data $\left(u_{0}, u_{1}\right)$ and $f$,

$$
E^{n+1}[u]\left(\Sigma_{R}^{-}\right) \lesssim\left\|u_{0}\right\|_{H^{n+1}}^{2}+\left\|u_{1}\right\|_{H^{n}}^{2}+\|f\|_{L E_{K}^{n *}}^{2}
$$

Thus it suffices to prove that for $\tilde{v}_{0}>0$ we have

$$
E^{n+1}[u]\left(\Sigma_{R}^{+}\right)+E^{n+1}[u]\left(\tilde{v}_{0}\right)+\|u\|_{L E_{K}^{n+1}}^{2} \lesssim E^{n+1}[u]\left(\Sigma_{R}^{-}\right)+\|f\|_{L E_{K}^{n *}}^{2}
$$

We will prove this for $n=2$, and the proof for the other cases will follow in a similar manner by induction.

Since $\partial_{\tilde{v}}$ is a Killing vector field, we have $\square_{K}\left(\partial_{\tilde{v}} u\right)=\partial_{\tilde{v}} f$. Then by Theorem 4.1 we obtain

$$
E\left[\partial_{\tilde{v}} u\right]\left(\Sigma_{R}^{+}\right)+E\left[\partial_{\tilde{v}} u\right]\left(\tilde{v}_{0}\right)+\left\|\partial_{\tilde{v}} u\right\|_{L E_{K}^{1}}^{2} \lesssim E^{2}[u]\left(\Sigma_{R}^{-}\right)+\|f\|_{L E_{K}^{1 *}}^{2}
$$


In order to control the rest of the second order derivatives we take advantage of the equation, which takes the form

$$
\left(g^{\tilde{v} \tilde{v}} \partial_{\tilde{v} \tilde{v}}+2 g^{\tilde{v} \tilde{\phi}} \partial_{\tilde{v} \tilde{\phi}}+L\right) u=f
$$

where $L$ is a spatial partial differential operator of order 2 . This is most useful in the region where $\partial_{\tilde{v}}$ is time-like. Given $\epsilon>0$, this happens in the region of the form $r>2 M+\epsilon$ provided that $a$ is sufficiently small. The fact that $\partial_{\tilde{v}}$ is time-like is equivalent to the ellipticity of the spatial part $L$ of $\square_{K}$. From (4.44) we obtain at $\tilde{v}=\tilde{v}_{0}$

$$
\|L u\|_{L^{2}\left(\Sigma_{v_{0}}\right)}^{2} \lesssim E[u]\left(v_{0}\right)+E\left[\partial_{\tilde{v}} u\right]\left(v_{0}\right)+\|f\|_{L^{2}\left(\Sigma_{v_{0}}\right)}^{2}
$$

The operator $L$ on the left is elliptic in $r \geq 2 M+\epsilon$, therefore by a standard elliptic estimate we obtain

$$
E[\nabla u]\left(\Sigma_{\tilde{v}_{0}} \cap\{r>2 M+\epsilon\}\right) \lesssim E[u]\left(v_{0}\right)+E\left[\partial_{\tilde{v}} u\right]\left(v_{0}\right)+\|f\|_{L^{2}\left(\Sigma_{v_{0}}\right)}^{2}
$$

A similar elliptic analysis leads to the corresponding local energy bound,

$$
\begin{aligned}
\|\nabla u\|_{L E_{K}^{1}\left(\mathcal{M}_{R} \cap\{r>2 M+2 \epsilon\}\right)}^{2} & \lesssim\|u\|_{L E_{K}^{1}\left(\mathcal{M}_{R}\right)}^{2}+\left\|\partial_{\tilde{v}} u\right\|_{L E_{K}^{1}\left(\mathcal{M}_{R}\right)}^{2}+\|f\|_{L^{2}\left(\mathcal{M}_{R}\right)}^{2} \\
& +E[\nabla u]\left(\Sigma_{0} \cap\{r>2 M+\epsilon\}\right)+E[\nabla u]\left(\Sigma_{\tilde{v}_{0}} \cap\{r>2 M+\epsilon\}\right)
\end{aligned}
$$

where the last two terms account for the output of integrations by parts in $\tilde{v}$.

We are left to deal with the case $r<2 M+2 \epsilon$, where $g^{r r}$ is small and simply using the equation (4.45) does not suffice. Let $\zeta(r)$ be a smooth cutoff function such that $\zeta=1$ on $\left[r_{e}, r_{+}+2 \epsilon\right]$ and $\zeta=0$ when $r>r_{+}+3 \epsilon$. Then we need bounds for the function $w=\zeta u$, which solves

$$
\square_{K} w=\zeta f+\left[\square_{K}, \zeta\right] u:=g
$$

The commutator above is supported in the region $\{2 M+2 \epsilon \leq r \leq 2 M+3 \epsilon\}$ where we already have good estimates for $u$. Recall that in the region $\{r<2 M+3 \epsilon\}$ the $L E_{K}^{1}$ and $L E_{K}^{*}$ norms are equivalent with the $H^{1}$, respectively $L^{2}$ norm. Hence it remains to prove that for all functions $w$ with support in $\{r<2 M+3 \epsilon\}$ which solve $\square_{K} w=g$ we have

$$
E[\nabla w]\left(\Sigma_{R}^{+}\right)+E[\nabla w]\left(\tilde{v}_{0}\right)+\|\nabla w\|_{H^{1}\left(\mathcal{M}_{R}\right)}^{2} \lesssim E^{2}[u]\left(\Sigma_{R}^{-}\right)+\|g\|_{H^{1}\left(\mathcal{M}_{R}\right)}^{2}
$$

This is an estimate which is localized near the event horizon, and we will prove it taking advantage of the red shift effect.

Since $\partial_{\tilde{v}}$ is Killing, this bound follows directly from Theorem 4.1 for the $\partial_{\tilde{v}} w$ component of $\nabla w$,

$$
E\left[\partial_{\tilde{v}} w\right]\left(\Sigma_{R}^{+}\right)+E\left[\partial_{\tilde{v}} w\right]\left(\tilde{v}_{0}\right)+\left\|\partial_{\tilde{v}} w\right\|_{H^{1}(D)}^{2} \lesssim E^{2}[w]\left(\Sigma_{R}^{-}\right)+\|g\|_{H^{1}(D)}^{2}
$$

Consider now the angular derivatives of $w, \partial_{\omega} w$. We know that

$$
\left[\square_{S}, \partial_{\omega}\right]=0
$$

since the Schwarzschild metric is spherically symmetric. Hence by (4.18) it follows that $\left[\square_{K}, \partial_{\omega}\right]$ is a second order operator whose coefficients have size $O(a)$. Hence by Theorem 4.1 we obtain

$$
E\left[\partial_{\omega} w\right]\left(\Sigma_{R}^{+}\right)+E\left[\partial_{\omega} w\right]\left(\tilde{v}_{0}\right)+\left\|\partial_{\omega} w\right\|_{H^{1}(D)} \lesssim E^{2}[w]\left(\Sigma_{R}^{-}\right)+\|g\|_{H^{1}(D)}^{2}+a\left\|\nabla_{\omega} w\right\|_{H^{1}(D)}^{2}
$$

We still need to bound $\partial_{r} w$. For that we compute the commutator

$$
\left[\square_{K}, \partial_{r}\right] w=-\left(\partial_{r} g^{r r}\right) \partial_{r r} w+T w
$$


where $T$ stands for a second order operator with no $\partial_{r}^{2}$ terms. The key observation, which is equivalent to the red shift effect, is that $\partial_{r} g^{r r}>0$ near $r=2 M$. Thus for $X, C$ and $q$ as in Lemma 4.2 we can write the equation for $\partial_{r} w$ in the form

$$
\left(\square_{K}-\gamma[(X+C K)+q]\right) \partial_{r} w=\partial_{r} g+T w
$$

with $T$ as above and most importantly, a positive coefficient $\gamma$. Because of this the operator

$$
B=\square_{K}-\gamma[(X+C K)+q]
$$

satisfies the same estimate in Theorem 4.1 as $\square_{g}$ for functions supported near the event horizon. Indeed, the same proof goes through as in Theorem 3.1. Writing the integral identity (4.21) for $w$ we see the contribution of $\gamma$ is negative, therefore we obtain the inequality

$$
\int_{D} Q^{K}[w, X, q, m] d V_{K} \leq-\int_{D}\left(\partial_{r} g+T w\right)((X+C K) w+q w) d V_{K}+B D R^{K}[w]
$$

By (4.23) the left hand side is positive definite for $r<2 M+3 \epsilon$. Using Cauchy-Schwarz for the first term on the right and (4.22) for the second we obtain

$$
E\left[\partial_{r} w\right]\left(\Sigma_{R}^{+}\right)+E\left[\partial_{r} w\right]\left(\tilde{v}_{0}\right)+\left\|\partial_{r} w\right\|_{H^{1}(D)}^{2} \lesssim E^{2}[w]\left(\Sigma_{R}^{-}\right)+\left\|\partial_{r} g+T w\right\|_{L^{2}(D)}^{2}
$$

Since $T$ contains no second order $r$ derivatives, this leads to

$$
E\left[\partial_{r} w\right]\left(\Sigma_{R}^{+}\right)+E\left[\partial_{r} w\right]\left(\tilde{v}_{0}\right)+\left\|\partial_{r} w\right\|_{H^{1}(D)}^{2} \lesssim E^{2}[w]\left(\Sigma_{R}^{-}\right)+\|g\|_{H^{1}(D)}+a\left\|\nabla_{\omega, \tilde{v}} w\right\|_{H^{1}(D)}^{2}
$$

Then the desired bound (4.46) follows by combining (4.47), (4.48) and (4.49) with appropriate coefficients.

As an easy corollary, one obtains from Sobolev embeddings the pointwise boundedness result,

Corollary 4.5. If $u$ satisfies $\square_{K} u=0$ in $\mathcal{M}_{R}$ with initial data $\left(u_{0}, u_{1}\right) \in H^{2} \times H^{1}$ in $\Sigma_{R}^{-}$, then

$$
\|u\|_{L^{\infty}} \lesssim\left\|u_{0}\right\|_{H^{2}}+\left\|u_{1}\right\|_{H^{1}}
$$

\section{REFERENCES}

[1] Serge Alinhac: Energy multipliers for perturbations of Schwarzschild metrics, preprint.

[2] Serge Alinhac: On the Morawetz-Keel-Smith-Sogge inequality for the wave equation on a curved background. Publ. Res. Inst. Math. Sci. 42(3) (2006), 705-720

[3] P. Blue and A. Soffer: Semilinear wave equations on the Schwarzschild manifold I: Local decay estimates. Adv. Differential Equations 8 (2003), 595-614.

[4] P. Blue and A. Soffer: The wave equation on the Schwarzschild metric II: Local decay for the spin-2 Regge-Wheeler equation. J. Math. Phys. 46 (2005), 9pp.

[5] P. Blue and A. Soffer: Errata for "Global existence and scatttering for the nonlinear Schrödinger equation on Schwarzschild manifolds", "Semilinear wave equations on the Schwarzschild manifold I: Local decay estimates", and "The wave equation on the Schwarzschild metric II: Local decay for the spin 2 Regge Wheeler equation", preprint.

[6] P. Blue and A. Soffer: Phase space analysis on some black hole manifolds, preprint.

[7] P. Blue and A. Soffer: Improved decay rates with small regularity loss for the wave equation about a Schwarzschild black hole, preprint. 
[8] P. Blue and J. Sterbenz: Uniform decay of local energy and the semi-linear wave equation on Schwarzschild space. Comm. Math. Phys. 268 (2006), 481-504.

[9] B. Carter: Global structure of the Kerr family of gravitational fields. Phys. Rev. 174 , (1968), 1559-1571.

[10] S. Chandrasekhar, The Mathematical Theory of Black Holes, volume 69 of The International Series of Monographs on Physics, (Clarendon Press, Oxford, U.K., 1983).

[11] M. Dafermos and I. Rodnianski: The red-shift effect and radiation decay on black hole spacetimes, preprint.

[12] M. Dafermos and I. Rodnianski: A note on energy currents and decay for the wave equation on a Schwarzschild background, preprint.

[13] M. Dafermos and I. Rodnianski: A proof of the uniform boundedness of solutions to the wave equation on slowly rotating Kerr backgrounds, preprint.

[14] F. Finster, N. Kamran, J. Smoller, S. T. Yau: Decay of solutions of the wave equation in Kerr geometry. Comm. Math. Phys. 264 (2006), 465 Û503

[15] S. W. Hawking and G. F. R. Ellis: The large scale structure of space-time. Cambridge Monographs on Mathematical Physics, No. 1. London, New York: Cambridge University Press 1973.

[16] C. E. Kenig, G. Ponce, and L. Vega: On the Zakharov and Zakharov-Schulman systems. J. Funct. Anal. 127 (1995), 204-234.

[17] M. Keel, H. Smith, and C. D. Sogge: Almost global existence for some semilinear wave equations. J. Anal. Math. 87 (2002), 265-279.

[18] I. Laba and A. Soffer: Global existence and scattering for the nonlinear Schrödinger equation on Schwarzschild manifolds. Helv. Phys. Acta 72(1999), 274-294.

[19] C. Morawetz: Time decay for the nonlinear Klein-Gordon equations. Proc. Roy. Soc. Ser. A. 306 (1968), 291-296.

[20] Jason Metcalfe and Christopher D. Sogge: Long-time existence of quasilinear wave equations exterior to star-shaped obstacles via energy methods. SIAM J. Math. Anal. 38(1)(2006), 188-209 (electronic)

[21] J. Marzuola, J. Metcalfe, D. Tataru, and M. Tohaneanu: Strichartz estimates on Schwarzschild black hole backgrounds, preprint.

[22] J. Metcalfe and D. Tataru: Global parametrices and dispersive estimates for variable coefficient wave equations, preprint.

[23] H. F. Smith and C. D. Sogge: Global Strichartz estimates for nontrapping perturbations of the Laplacian. Comm. Partial Differential Equations 25 (2000), 2171-2183.

[24] J. Sterbenz: Angular regularity and Strichartz estimates for the wave equation. With an appendix by I. Rodnianski. Int. Math. Res. Not. 2005, 187-231.

[25] W. A. Strauss: Dispersal of waves vanishing on the boundary of an exterior domain. Comm. Pure Appl. Math. 28 (1975), 265-278.

[26] Volker Perlick, Gravitational Lensing from a Spacetime Perspective. Living Rev. Relativity bf 7 , (2004), 9. URL (cited on 10/01/2008): http://www.livingreviews.org/lrr-2004-9

Department of Mathematics, University of California, Berkeley, CA 94720-3840

Department of Mathematics, University of California, Berkeley, CA 94720-3840 